\title{
The Key Role of the Latent N-H Group in Milstein's Catalyst for Ester Hydrogenation
}

\author{
John Pham, Cole E. Jarczyk, Eamon F. Reynolds, Sophie. E. Kelly, Thao Kim, Tianyi He, Jason M. Keith*, \\ and Anthony R. Chianese* \\ Department of Chemistry, Colgate University, 13 Oak Drive, Hamilton, New York 13346, United States
}

\begin{abstract}
We previously demonstrated that Milstein's seminal diethylamino-substituted PNN-pincerruthenium catalyst for ester hydrogenation is activated by dehydroalkylation of the pincer ligand, releasing ethane and eventually forming an NHEt-substituted derivative that we proposed is the active catalyst. In this paper, we present a computational and experimental mechanistic study supporting this hypothesis. Our DFT analysis shows that the minimum-energy pathways for hydrogen activation, ester hydrogenolysis, and aldehyde hydrogenation rely on the key involvement of the nascent $\mathrm{N}-\mathrm{H}$ group. We have isolated and crystallographically characterized two catalytic intermediates, a ruthenium dihydride and a ruthenium hydridoalkoxide, the latter of which is the catalyst resting state. A detailed kinetic study shows that catalytic ester hydrogenation is first-order in ruthenium and hydrogen, shows saturation behavior in ester, and is inhibited by the product alcohol. A global fit of the kinetic data to a simplified model incorporating the hydridoalkoxide and dihydride intermediates and three kinetically relevant transition states showed excellent agreement with the results from DFT.
\end{abstract}

\section{Introduction}

Catalytic transformations relying on metal-ligand-cooperative hydrogenation or dehydrogenation of polar substrates have seen a dramatic expansion in development over the past decade and a half, following the disclosure by Milstein and co-workers of the PNN-pincer-ruthenium complex RuPNN ${ }^{\text {dearom }}$ (Scheme 1), shown to be active under relatively mild conditions for the hydrogenation of esters to alcohols, ${ }^{1}$ as well as the reverse reaction, the acceptorless dehydrogenative coupling (ADC) of primary alcohols to esters. ${ }^{2}$ This complex has since been applied to a wide range of mechanistically related transformations. ${ }^{3}$ In the original reports, the dearomatized complex RuPNN ${ }^{\text {dearom }}$ was shown to react reversibly with hydrogen at room temperature to give the rearomatized dihydride complex RuPNN ${ }^{\mathbf{H 2}}$. Based on this observation, a mechanism was proposed that involved this heterolytic cleavage of hydrogen as a key step in catalytic ester hydrogenation.

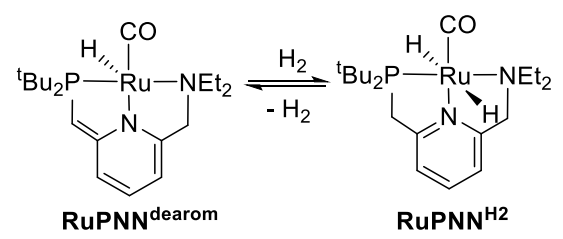

Scheme 1. Reversible activation of hydrogen mediated by RuPNN ${ }^{\text {dearom }}$. 
In the years since the initial reports on RuPNN ${ }^{\text {dearom }}$, many researchers have studied the effect of catalyst structure on activity in ester hydrogenation, and several highly optimized catalysts have been discovered that give more than 10,000 turnovers at full substrate conversion. ${ }^{4}$ Common to almost all of these elite catalysts is an $\mathrm{N}-\mathrm{H}$ functional group on the ligand. In some cases, the $\mathrm{N}-\mathrm{H}$ group was demonstrated to be essential for high catalytic activity through the synthesis of control ligands where $\mathrm{N}$ $\mathrm{H}$ was replaced with $\mathrm{N}-\mathrm{Me}, \mathrm{N}-\mathrm{Bn}$, or $\mathrm{O} .{ }^{4 a}, 4 \mathrm{c}, 4 \mathrm{e}$ In many cases, minimum-energy pathways involving deprotonation of the N-H group have been identified through density functional theory, $4 c, 4 f, 4 h, 4 i, 5$ although recent computational work has suggested that in some cases, the $\mathrm{N}-\mathrm{H}$ group may function in catalysis as a hydrogen-bond donor without being deprotonated on the catalytic cycle. ${ }^{5 b, 6}$ Although many of the most highly active catalysts for ester hydrogenation feature an $\mathrm{N}-\mathrm{H}$ functional group, several structural motifs lacking an $\mathrm{N}-\mathrm{H}$ group have also been reported. In particular, several ruthenium catalyst variants instead featuring dialkylamino side groups like RuPNN ${ }^{\text {dearom }}$ have shown activity. ${ }^{4 d, 7}$

During mechanistic studies of our previously reported ${ }^{7 c, 7 d, 8} \mathrm{CNN}$-pincer-ruthenium catalysts for ester hydrogenation, we made a surprising observation: precatalysts featuring $\mathrm{NEt}_{2}$ or N$^{\mathrm{P}} \mathrm{Pr}_{2}$ side groups underwent an unexpected dehydroalkylation reaction, releasing an equivalent of ethane or propane early on in catalytic reactions. ${ }^{9}$ The observation of catalytic induction periods concomitant with the release of alkane established that dehydroalkylation was a necessary step in formation of the active catalyst. Milstein's catalyst RuPNN ${ }^{\text {dearom }}$, which features an $\mathrm{NEt}_{2}$ side group, also showed an induction period for ester hydrogenation, and released ethane concomitantly with the onset of catalytic activity. By heating our CNN- and Milstein's PNN-pincer precatalysts in the presence of tricyclohexylphosphine, we were able to trap the intermediate products of dehydroalkylation as five-coordinate ruthenium( 0 ) complexes, where the dialkylamino side group was transformed to an imine functionality (PNN variant shown in Scheme 2). RuPNN ${ }^{\text {imine }}$, the ruthenium(0) derivative of RuPNN ${ }^{\text {dearom }}$, is dramatically more active as a precatalyst for ester hydrogenation than its precursor, and is by some measures the most efficient catalyst currently known for ester hydrogenation, giving in excess of 10,000 catalytic turnovers at room

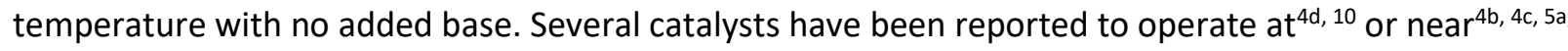
${ }^{11}$ room temperature, but require significant quantities of strongly basic additives such as $\mathrm{NaO}^{\mathrm{t}} \mathrm{Bu}$ and $\mathrm{KO}^{\mathrm{t}} \mathrm{Bu}$. Conversely, several catalysts operate without the need for added base, but require temperatures of $80^{\circ} \mathrm{C}$ or higher..$^{1,58,12}$

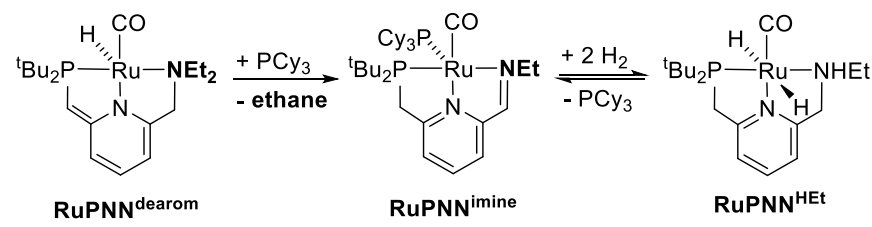

Scheme 2. Dehydroalkylation of Milstein's catalyst and reversible $\mathrm{H}_{2}$ addition giving RuPNN ${ }^{\text {HEt }}$.

To further probe the catalyst speciation under operating conditions, we monitored the reaction of RuPNN ${ }^{\text {imine }}$ with hydrogen at room temperature. Under 10 bar $\mathrm{H}_{2}$, RuPNN ${ }^{\text {imine }}$ converts quantitatively to the dihydride complex RuPNN ${ }^{\mathrm{HEt}}$, involving a net hydrogenation of the imine functional group and a net oxidative addition of $\mathrm{H}_{2}$ to the ruthenium center (Scheme 2). As RuPNN ${ }^{\text {HEt }}$ contains a ruthenium hydride 
and $\mathrm{N}-\mathrm{H}$ group, we proposed ${ }^{9}$ that it may be an active catalytic intermediate in reactions initiated by RuPNN $^{\text {imine }}$ and RuPNN ${ }^{\text {dearom }}$, operating by a metal-ligand-cooperative mechanism analogous to that proposed for other elite $\mathrm{N}-\mathrm{H}$-containing catalysts.

The discovery of the dehydroalkylative activation of RuPNN $\mathbf{N}^{\text {dearom }}$ has potentially broad mechanistic implications. Because we have demonstrated that RuPNN ${ }^{\text {dearom }}$ is not a kinetically competent intermediate and must undergo dehydroalkylation prior to being catalytically active for ester hydrogenation, it is unlikely that the originally proposed mechanism ${ }^{1}$ is correct. Three reports in the literature apply density functional theory to predict the mechanism of ester hydrogenation or the reverse ADC, catalyzed by RuPNN ${ }^{\text {dearom }} \cdot{ }^{13}$ Since these studies rely on the catalytic intermediacy of RuPNN ${ }^{\text {dearom }}$ or RuPNN ${ }^{\mathrm{H2}}$, they too are unlikely to be correct. More broadly, RuPNN ${ }^{\text {dearom }}$ has been applied as a catalyst for a wide range of related hydrogenation and dehydrogenation reactions, including amine-alcohol coupling, ${ }^{3 a-d}$ couplings of amines ${ }^{3 e}$ or alcohols ${ }^{3 f}$ with esters, organic carbonate hydrogenation, ${ }^{3 \mathrm{~g}}$ carbon dioxide hydrogenation, ${ }^{3 \mathrm{~h}}$ and amide $\alpha$-alkylation with alcohols. ${ }^{3 \mathrm{i}}$ All of these transformations are conducted at or above $100^{\circ} \mathrm{C}$, where the dehydroalkylation reaction occurs rapidly $\left(\mathrm{t}_{1 / 2}=6 \mathrm{~min}\right.$ at $\left.100^{\circ} \mathrm{C}\right)$. As such, it is possible that RuPNN ${ }^{\text {dearom }}$ is inactive prior to dehydroalkylation in these systems as well, which may call into question the DFT studies of these transformations. ${ }^{13 a, 14}$

Kinetic studies have the potential to validate or falsify the findings from computation, as they show conclusively what species are consumed or released on the pathway from the turnover-frequencydetermining intermediate (TDI) to the turnover-frequency-determining-transition state (TDTS). ${ }^{15}$ For example, recent kinetic investigations of metal-catalyzed hydroformylation ${ }^{16}$ and ketone hydrogenation ${ }^{17}$ have provided deep insight into the underlying reaction mechanisms. Although catalytic ester hydrogenation and its microscopic reverse, $A D C$, have been studied intensively through computational methods, detailed experimental mechanistic investigations, especially kinetic studies, are scarce. In studies of an iridium-bipyridine catalyst system, Brewster, Sanford, and Goldberg determined the dependence of turnover number at low conversion on the concentrations of catalyst, hydrogen, and ester. ${ }^{12 \mathrm{~h}}$ However, as reactions were not monitored over time, this study did not probe for potential activation of catalyst observed as an induction period, and did not probe for potential product inhibition. Filonenko, Pidko and coworkers reported time-course studies of ester hydrogenation catalyzed by CNC-pincer-ruthenium complexes, but did not determine the detailed dependences of the rate on concentrations..$^{4 \mathrm{e}} \mathrm{O}$ and Morris also reported time-course studies for ester hydrogenation catalyzed by ruthenium complexes of NHC-amine ligands, but also did not determine a rate law. ${ }^{5 a}$

In this paper, we describe a computational and experimental mechanistic study of ester hydrogenation catalyzed by RuPNN ${ }^{\text {imine }}$. We report the crystallographic characterization of the key dihydride intermediate RuPNN ${ }^{\mathrm{HEt}}$, and the synthesis and characterization of the ruthenium-hydridoalkoxide RuPNN ${ }^{\text {HOEt }}$, which represents the catalytic resting state and TDI. Detailed kinetic studies show an induction period at room temperature during which RuPNN ${ }^{\text {imine }}$ is converted to the active form, and after which the reaction shows first-order dependence on [catalyst] and [hydrogen], first-order saturation behavior in [ester], and a transition from inverse second-order to inverse-first-order inhibition by the product alcohol. All of this kinetic behavior, as well as the overall rate of reaction, is consistent with the minimum-energy pathway calculated using density functional theory. 


\section{Computational Mechanistic Analysis}

Background. Previous computational studies of the mechanism of ester hydrogenation catalyzed by transition-metal complexes with a pendant $\mathrm{N}-\mathrm{H}$ functional group have converged on a bicyclic pathway, ${ }^{4 c, 4 f, 4 h, 4 i, 5}$ which can be separated into three linear sequences (Scheme 3 ). In the hydrogenactivation sequence, the $\mathrm{H}-\mathrm{H}$ bond is cleaved heterolytically, placing a hydride on the metal center and a proton on the basic nitrogen center. In ester hydrogenolysis, these hydrogen atoms are transferred to the ester substrate, and cleavage of the $\mathrm{C}-\mathrm{O}$ bond facilitated by the catalyst produces an aldehyde intermediate and one product alcohol molecule. In the aldehyde-hydrogenation sequence, the intermediate aldehyde is reduced to alcohol by the hydrogenated form of the catalyst. In one important variation on this scheme, it is possible that the nitrogen remains protonated throughout catalysis if an exogenous alkoxide base participates in hydrogen cleavage directly. ${ }^{5 b, 6}$

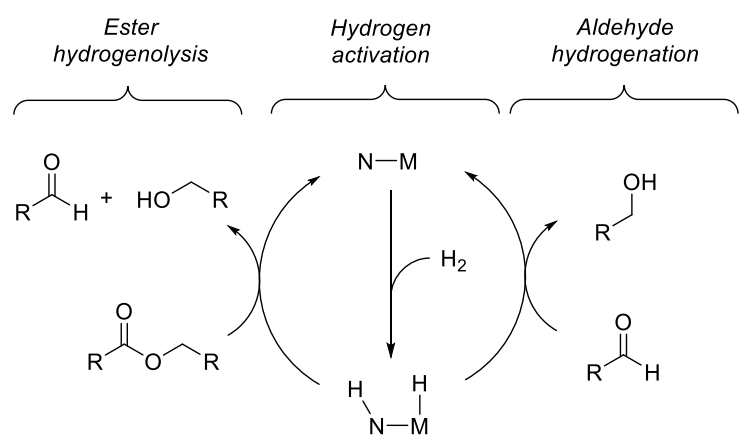

Scheme 3. Two linked catalytic cycles for ester hydrogenation

Pathway for Hydrogen Activation. We chose ethyl acetate as an appropriate model ester for computational study, for several reasons: 1 ) its use is well-precedented in both experimental and computational work; 2 ) it is small enough to minimize issues resulting from a large number of potential conformations; 3 ) it is large enough to appropriately model the steric interactions of common ester substrates with the catalyst. In particular, we expected the energetics of ethyl acetate hydrogenation to closely mimic those of hexyl hexanoate, which we employed in the kinetic studies described below.

We began by probing a range of possible pathways for the activation of $\mathrm{H}_{2}$, informed by the rich history of prior work on related reactions. Our search for the MEP for $\mathrm{H}_{2}$ activation covered metalligand-cooperative heterolytic cleavage involving the $\mathrm{N}-\mathrm{H}$ functional group, as proposed by Noyori and co-workers for their seminal carbonyl hydrogenation catalysts, ${ }^{18}$ both with and without explicit ethanol molecules to serve as proton shuttles. Noyori-type mechanisms for $\mathrm{H}_{2}$ activation have been identified for a range of catalysts for ester hydrogenation or ADC. ${ }^{4 c, 4 f, 4 h, 4 i, 5 a, 5 c-j, 6 \mathrm{~d}}$ We also carefully searched for pathways involving cooperative activation of $\mathrm{H}_{2}$ through the ruthenium center and an ethoxide anion, which Dub et al. showed can bypass the deprotonation of the $\mathrm{N}-\mathrm{H}$ group on the pincer ligand. ${ }^{5 b, 6}$ Lastly, we exhaustively examined pathways for $\mathrm{H}_{2}$ activation involving deprotonated $\mathrm{CH}_{2}$ linkers on the pincer ligand, as originally proposed by Milstein and coworkers ${ }^{1}$ and identified by DFT in many studies. ${ }^{13 a, 14 a-e,}$ $14 \mathrm{~g}, 19$ 
Figure 1 shows the pathway we identified with the lowest overall barrier, which we describe as a "proton brigade" because of the involvement of two ethanol molecules in the stepwise cleavage of the $\mathrm{H}-\mathrm{H}$ bond. Beginning with the ethanol-stabilized hydridoalkoxide species a2, whose experimental

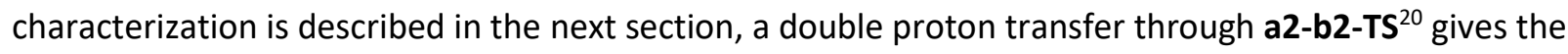
$\mathrm{N}$-deprotonated species $\mathbf{b 2}$ with a neutral ethanol molecule coordinated to Ru. Then, this ethanol dissociates to give the unsaturated species $\mathbf{c 2}$, which transfers a proton back to nitrogen to give $\mathbf{d} \mathbf{2}$ before binding $\mathrm{H}_{2}$ in the $\sigma$-complex $\mathbf{f 2}$, in which the nitrogen is protonated and an ethoxide anion is stabilized by two hydrogen bonds. Then, $\mathrm{H}_{2}$ is cleaved through the proton-shuttle transition state f2-g2TS, resulting in the dihydride species $\mathbf{g} 2$ (with two associated ethanol molecules) or g1 (with one associated ethanol). Although this pathway does involve temporary deprotonation of nitrogen between a2 and $\mathbf{d} \mathbf{2}$, the reformation of the $\mathrm{N}-\mathrm{H}$ bond is not concerted with $\mathrm{H}_{2}$ cleavage. We also located a direct pathway connecting $\mathbf{2} \mathbf{2}$ to $\mathbf{d} \mathbf{2}$, where the Ru-bound ethoxide dissociates and deprotonates the outersphere ethanol molecule in a concerted manner through a2-d2-TS (Figure 2). This direct pathway, which keeps the $\mathrm{N}-\mathrm{H}$ bond intact for the entire $\mathrm{H}_{2}$-activation sequence, has a slightly higher barrier of 14.1 $\mathrm{kcal} / \mathrm{mol}$ as compared to $12.9 \mathrm{kcal} / \mathrm{mol}$ in the first pathway.

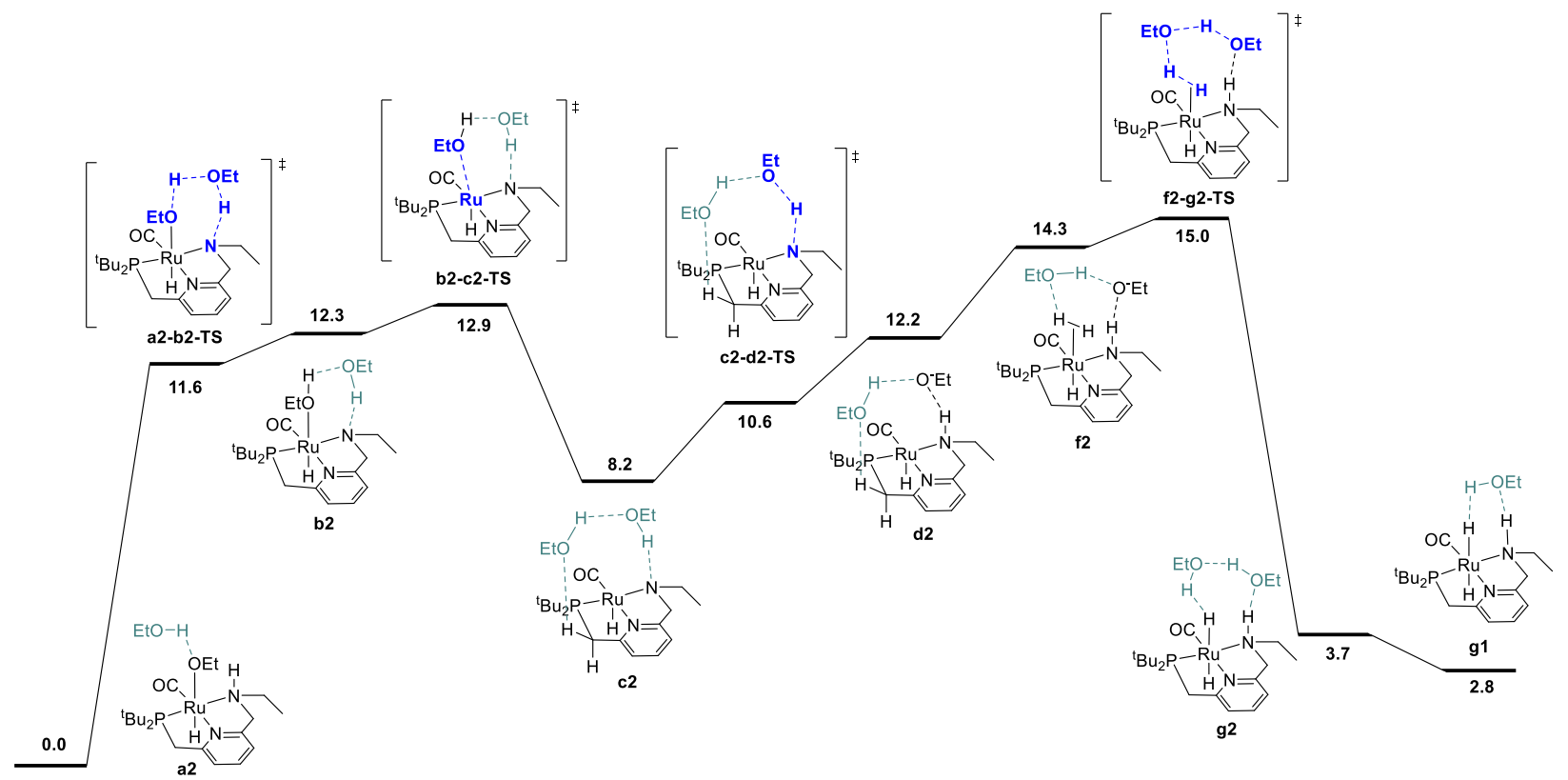

Figure 1. Minimum-energy pathway for hydrogen activation to convert the hydrido-ethoxide resting state $\mathbf{a} \mathbf{2}$ into dihydride intermediate $\mathbf{g} \mathbf{1}$. Throughout this work, atoms in bold and blue represent those atoms principally involved in bond-breaking and bond-forming events in transition states. Atoms shown in turquoise represent neutral ethanol molecules interacting with the main fragment through hydrogen bonds. Energies given represent standard-state free energies in $\mathrm{kcal} / \mathrm{mol}$ at $298.15 \mathrm{~K}$, relative to a2 and the organic reactants unless otherwise stated. 


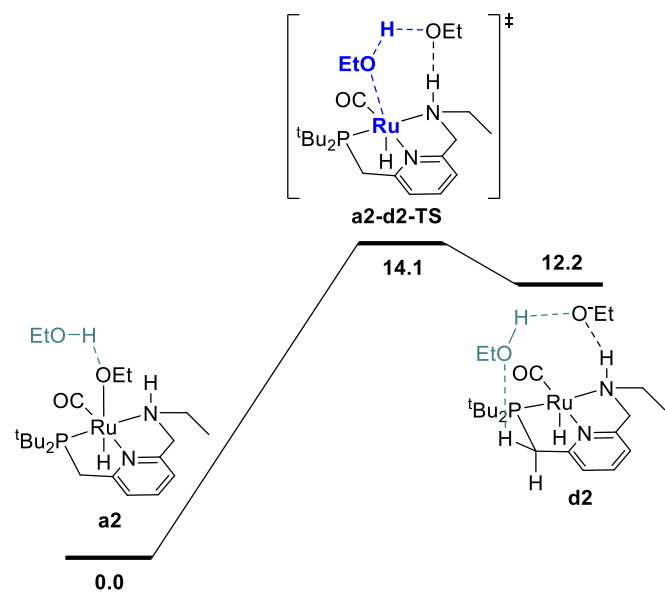

Figure 2. Alternate pathway to convert hydrido-ethoxide a2 into unsaturated intermediate $\mathbf{d} \mathbf{2}$, by direct dissociation of the ethoxide ligand without prior deprotonation of nitrogen.

In the MEP featured above in Figure 1, nitrogen is protonated prior to $\mathrm{H}-\mathrm{H}$ cleavage in the conversion of $\mathbf{c} \mathbf{2}$ to $\mathbf{f 2}$. We also identified a pathway where hydrogen coordination precedes reprotonation of nitrogen, which proceeds through a higher barrier of $15.2 \mathrm{kcal} / \mathrm{mol}$ (Figure 3 ).

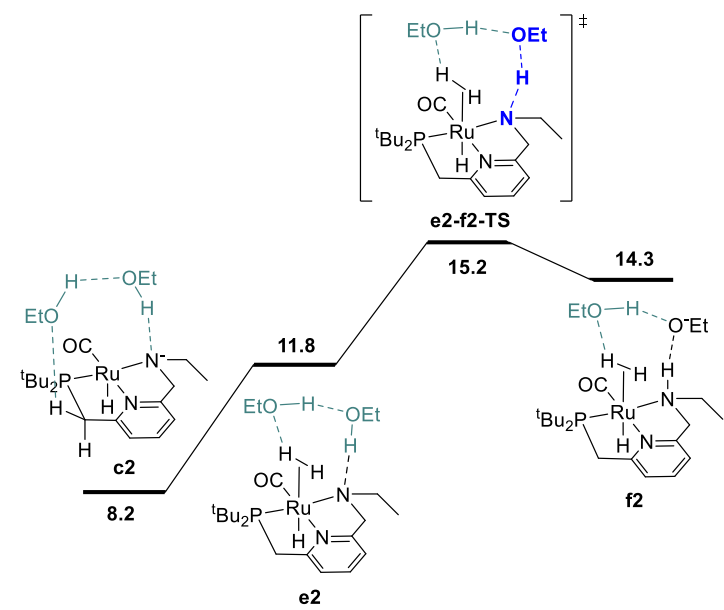

Figure 3. Alternate pathway to convert unsaturated intermediate $\mathbf{c} \mathbf{2}$ into dihydrogen complex $\mathbf{f 2}$, where dihydrogen coordination to give $\mathbf{e} \mathbf{2}$ precedes protonation of nitrogen to give $\mathbf{f 2}$.

All of the pathways shown above require passing through $\mathbf{f 2 - g 2 - T S}$ at $15.0 \mathrm{kcal} / \mathrm{mol}$ in the pathway for hydrogen activation. Notably, these proton-brigade pathways rely on the inclusion of two explicit ethanol molecules, both for the low overall barrier and for the stepwise proton-shuttle mechanisms. For comparison, we calculated analogous pathways involving only one ethanol molecule as proton shuttle and involving no ethanol molecules. These pathways, both concerted, are described in detail in the Supporting Information, and were found to proceed through higher overall barriers of 18.6 and 25.3 $\mathrm{kcal} / \mathrm{mol}$, respectively. 
We also searched exhaustively for pathways involving the activation of $\mathrm{H}_{2}$ mediated by deprotonated $\mathrm{CH}_{2}$ linkers of the pincer ligand, both with and without including explicit ethanol molecules as proton shuttles. These pathways, described in detail in the Supporting Information, would implicate dearomatized species similar to RuPNN ${ }^{\text {dearom }}$ as key intermediates in catalysis. All mechanisms of this nature were found to proceed through higher barriers for $\mathrm{H}_{2}$ cleavage, with a lowest identified barrier of $25.6 \mathrm{kcal} / \mathrm{mol}$ for mechanisms involving the $\mathrm{NCH}_{2}$ linker and $23.7 \mathrm{kcal} / \mathrm{mol}$ for the $\mathrm{PCH}_{2}$ linker. In summary, our work shows that the presence of the $\mathrm{N}-\mathrm{H}$ functional group is essential for activation of hydrogen with a low barrier. The $\mathrm{N}-\mathrm{H}$ group is temporarily deprotonated in our MEP, but a pathway where the $\mathrm{N}-\mathrm{H}$ group remains protonated and instead serves to stabilize intermediates and transition states through hydrogen-bonding is energetically similar, and cannot be excluded by DFT. Pathways involving deprotonation of $\mathrm{CH}_{2}$ linkers have significantly higher barriers and can be excluded.

Pathway for Ester Hydrogenolysis. The ester hydrogenolysis portion of the catalytic cycle involves the hydrogenation of the carbonyl functional group and cleavage of the $\mathrm{C}-\mathrm{O}$ bond, ultimately releasing one alcohol product molecule and an aldehyde intermediate. Prior work on many systems has identified the transfer of hydride from the metal to the carbonyl carbon as a key initial step, which generates a hemiacetaloxide intermediate. Two principal pathways for cleavage of the C-OEt bond have emerged, which have been shown to have similar barriers for related catalysts. These pathways differ by the coordination of either the aldehyde oxygen ${ }^{4 c, 4 f, 4 i, 5 d, 5 f, 5 h, 6 d}$ or the alkoxide oxygen ${ }^{4 h, 5 a, 5 h, 5 i, 6 d, 21}$ to Ru during C-O cleavage. In our system, we find these mechanisms have nearly identical barriers, as described below. The pathway shown in Figure 4 is an example of the former mechanism. Beginning from the dihydride intermediate $\mathbf{g 1}$, the ester replaces the hydrogen-bonded alcohol molecule to give the reactant complex $\mathbf{h}$, which transfers hydride from $\mathrm{Ru}$ to $\mathrm{C}$ to give the $\mathrm{C}-\mathrm{H} \sigma$-complex $\mathbf{i}$, where the hemiacetaloxide oxygen is stabilized by hydrogen-bonding to the $\mathrm{N}-\mathrm{H}$. Then, proton transfer from $\mathrm{N}$ to $\mathrm{O}$ gives $\mathbf{j}$, ${ }^{20}$ which rearranges to place the hydroxyl oxygen on $\mathrm{Ru}$ in $\mathbf{k}$, followed by reprotonation of nitrogen and subsequent hydrogen bond formation to give the Ru-bound hemiacetaloxide complex $\mathbf{m}$. Then, cleavage of the $\mathrm{C}-\mathrm{O}$ bond, concerted with proton transfer from $\mathrm{N}$ back to $\mathrm{O}$, gives $\mathbf{n}$, a looselybound complex of the product ethanol and intermediate acetaldehyde. Replacement of the aldehyde with another ethanol molecule gives $\mathbf{c 2}$, which connects back to the hydrogen activation pathway in Figure 1 and completes the first hydrogenation cycle. 


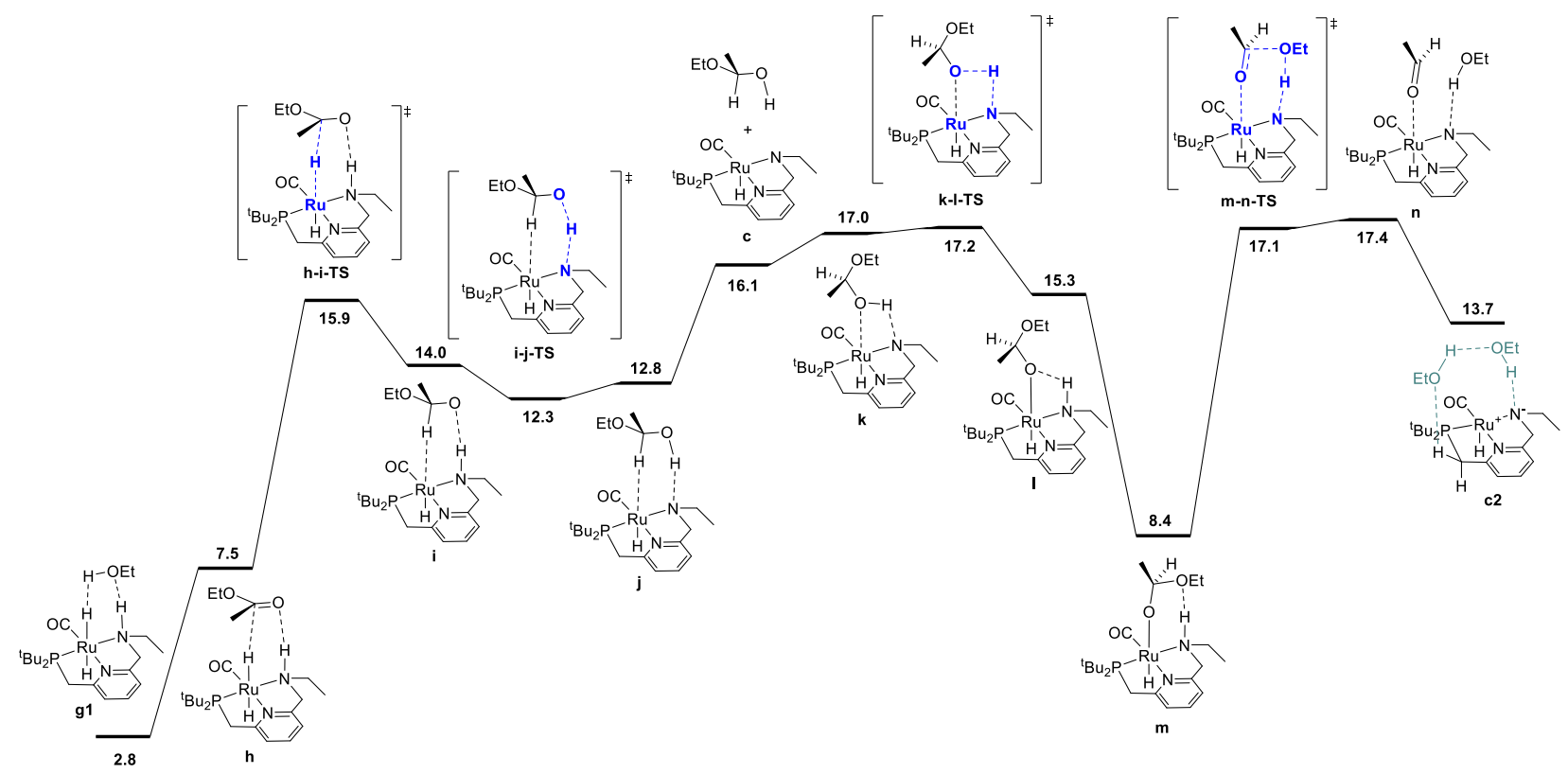

Figure 4. Minimum-energy pathway for hydrogenolysis of ethyl acetate to acetaldehyde and ethanol, accompanied by conversion of dihydride intermediate $\mathbf{g} \mathbf{1}$ into unsaturated intermediate $\mathbf{c 2}$, which connects back to the hydrogen-activation pathway shown in Figure 1. Note that the standard-state free energy of $13.7 \mathrm{kcal} / \mathrm{mol}$ reported here for $\mathbf{c} 2$ corresponds to release of acetaldehyde and binding of ethanol from $\mathbf{n}$, whereas the free-energy of $8.2 \mathrm{kcal} / \mathrm{mol}$ reported for $\mathbf{c} \mathbf{2}$ in Figure 1 is calculated against the ethyl acetate and dihydrogen reactants.

The ester hydrogenolysis pathway shown above proceeds through a highest barrier of 17.4 $\mathrm{kcal} / \mathrm{mol}$, which is the free energy of the intermediate species $\mathbf{n}$. Notably, intermediates $\mathbf{k}$ and $\mathbf{n}$ and transition states $\mathbf{k}-\mathbf{I}-\mathbf{T S}$ and $\mathbf{m}-\mathbf{n}$-TS all have essentially identical free energies of $17.0-17.4 \mathrm{kcal} / \mathrm{mol}$. Thus, flux through this sequence is limited by both the cleavage of the $\mathrm{O}-\mathrm{H}$ bond in $\mathbf{k}$ (essentially barrierless in the forward direction) and the cleavage of the $\mathrm{C}-\mathrm{O}$ bond in $\mathbf{m}$ (essentially barrierless in the reverse direction). ${ }^{20}$

We identified a different pathway for C-O cleavage, with a nearly identical overall barrier of 18.1 $\mathrm{kcal} / \mathrm{mol}$, which directly places the newly formed ethoxide rather than the aldehyde on ruthenium (Figure 5). Similar to the transformation identified by Hasanayn and termed a hydride-alkoxide metathesis, ${ }^{21}$ the hemiacetal $\sigma$-complex $\mathbf{j}$ rotates through $\mathbf{j}$-o-TS to place the ethoxy oxygen on $\mathrm{Ru}$ in $\mathbf{o}$. Then, proton transfer from $\mathrm{O}$ to $\mathrm{N}$ gives $\mathbf{p}$, and transfer of the ethoxy group from carbon to ruthenium through $\mathbf{p}$ - $\mathbf{q}$-TS gives the ethoxide complex $\mathbf{q}$, where the intermediate aldehyde is loosely associated with the $\mathrm{N}-\mathrm{H}$ through a hydrogen bond. Replacement of the aldehyde with a hydrogen-bonded ethanol molecule regenerates a2, completing the first hydrogenation cycle. As the overall barrier of 18.1 $\mathrm{kcal} / \mathrm{mol}$ for this pathway is nearly identical to the overall barrier of $17.4 \mathrm{kcal} / \mathrm{mol}$ for the pathway shown in Figure 4, our results do not unambiguously identify one pathway as preferred over the other, and it is possible that both ways operate in parallel. As an identical rate law would be predicted for either pathway, the kinetic data presented below do not distinguish between these pathways. 


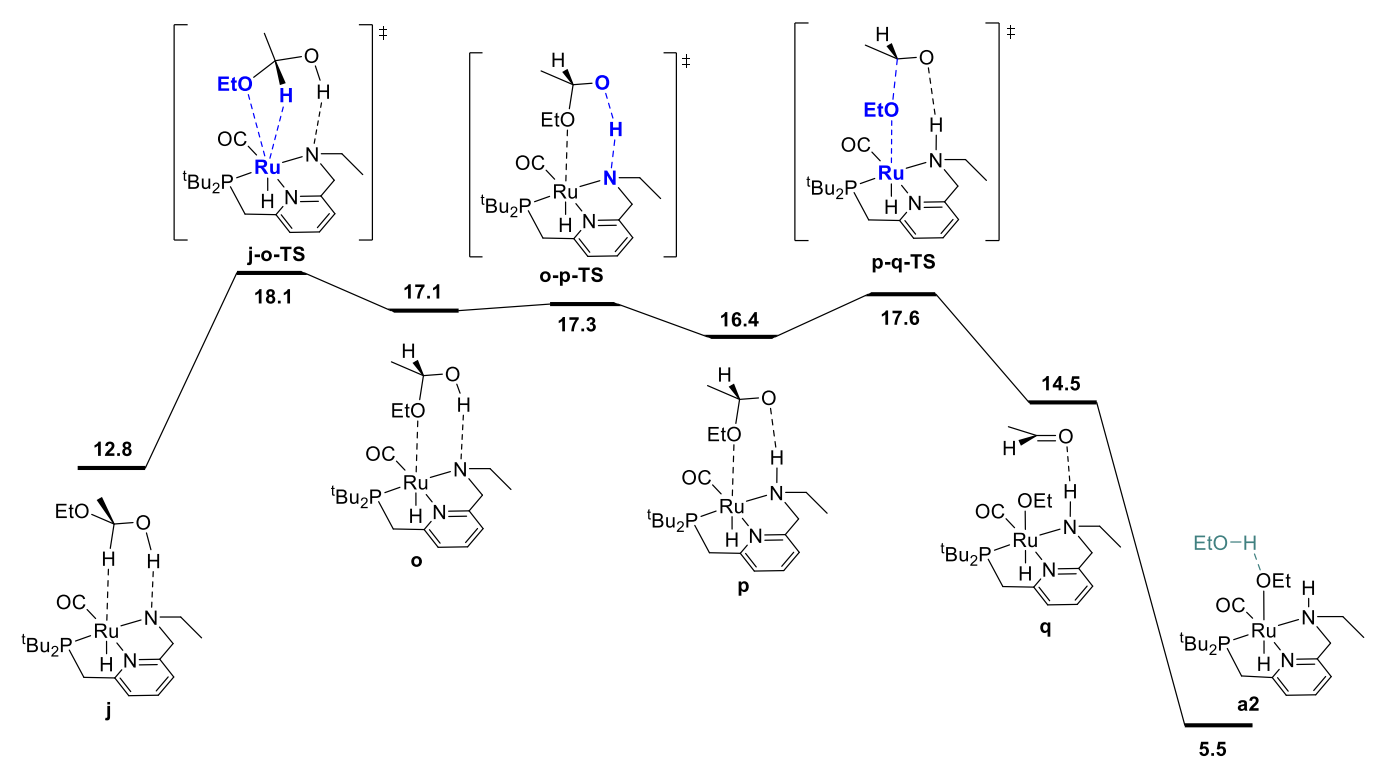

Figure 5. Alternative pathway for the conversion of hemiacetal complex $\mathbf{j}$ into hydrido-ethoxide $\mathbf{a} \mathbf{2}$ with release of acetaldehyde, following a hydride-ethoxide metathesis pathway as proposed by Hasanayn.

In Hasanayn's pathway for hydride-alkoxide metathesis, the ligand $\mathrm{C}-\mathrm{H}$ or $\mathrm{N}-\mathrm{H}$ remains protonated throughout ester hydrogenolysis, ${ }^{21}$ although the $\mathrm{N}-\mathrm{H}$ is temporarily deprotonated in both of our identified mechanisms. We examined a direct pathway for the conversion of the hemiacetaloxide intermediate $\mathbf{i}$ to $\mathbf{p}$ through a metathesis mechanism where the $\mathrm{N}-\mathrm{H}$ remains protonated, and find a slightly higher barrier of $21.4 \mathrm{kcal} / \mathrm{mol}$ (Figure 6), as compared to the $18.1 \mathrm{kcal} / \mathrm{mol}$ barrier for the pathway in Figure 5 involving the neutral hemiacetal and a deprotonated nitrogen.

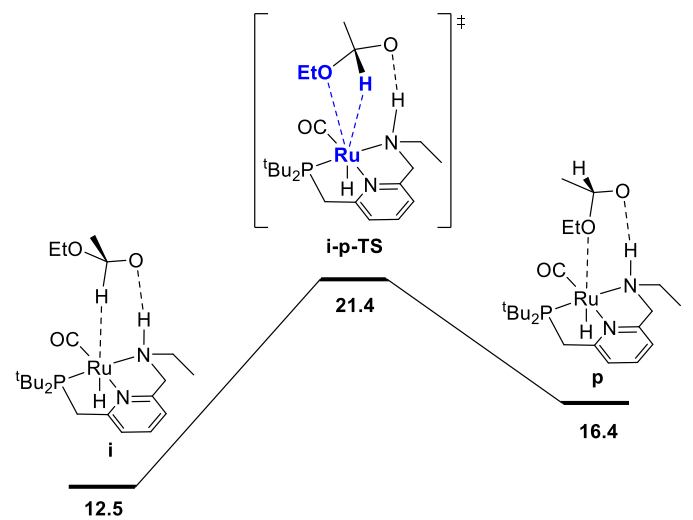

Figure 6. Alternative pathway for the conversion of $\mathrm{C}-\mathrm{H} \sigma$-complex $\mathbf{i}$ into O-complex $\mathbf{p}$, following a metathesis pathway analogous to that shown in Figure 5 without prior deprotonation of nitrogen. 
In the process of establishing the twin minimum-energy pathways described above in Figures 4 and 5 , we characterized diastereomeric sequences where the ester initially coordinates to Ru through the opposite face, and pathways involving an explicit ethanol molecule. As described in detail in the Supporting Information, we found slightly higher overall barriers for the diastereomeric pathways and similar overall barriers for pathways involving an explicit ethanol molecule. We also calculated a ruthenium-free pathway for the conversion of the hemiacetal to ethanol and acetaldehyde, and find a much higher barrier of $36.4 \mathrm{kcal} / \mathrm{mol}$, in line with previous work. ${ }^{6 \mathrm{~d}, 14 \mathrm{~h}}$ In summary, we find that ester hydrogenolysis proceeds in our system by well-precedented mechanisms for ruthenium-pincer catalysts possessing an $\mathrm{N}-\mathrm{H}$ group, and that the decomposition of the hemiacetal is mediated by the rutheniumpincer catalyst.

Pathway for Aldehyde Hydrogenation. The final portion of the catalytic cycle involves hydrogenation of the aldehyde intermediate to give the second equivalent of alcohol product. This sequence, as mediated by a ruthenium hydride complex with a pendent $\mathrm{N}-\mathrm{H}$ functional group on the ligand, has been studied extensively through DFT in the context of ester hydrogenation, but also has a longer history dating back to the original Noyori catalysts, which were highly efficient for aldehyde and ketone hydrogenation. ${ }^{18}$ For ester hydrogenation catalysts, the aldehyde hydrogenation step is generally found to have a lower barrier than the ester hydrogenolysis step, which along with the thermodynamic instability of the aldehyde with respect to reactants, is consistent with the lack of buildup of aldehyde in catalytic reactions. For our catalytic system, we identified the pathway shown in Figure 7, beginning with coordination of the aldehyde to form $\mathbf{r}$. This is followed by stepwise transfer of hydride and proton to the substrate from the ruthenium and nitrogen centers, respectively, giving intermediates $\mathbf{s}$ and $\mathbf{t}$. Dissociation of the $\mathrm{C}-\mathrm{H}$ $\sigma$-complex gives $\mathbf{c 1}$, which connects back to the hydrogen activation pathway.

In some past studies, ${ }^{6,13 c, 22}$ proton transfer from the ligand to the alkoxide oxygen was calculated to have a higher barrier than proton transfer from an exogenous alcohol molecule, which enables the construction of a pathway for hydrogenation where the ligand $\mathrm{N}-\mathrm{H}$ group (or $\mathrm{CH}_{2}$ linker) is never deprotonated. Pathways like this may have been missed in earlier work because of the optimization of structures without a solvent model, which can favor concerted proton/hydride transfer pathways and disfavor ion-pair intermediates such as $\mathbf{s}$. In our work, conducting geometry optimizations using a toluene continuum solvent model allowed the identification of the intermediate $\mathbf{s}$. As proton transfer from $\mathrm{N}$ to $\mathrm{O}$ through s-t-TS is barrierless ${ }^{20}$ and strongly exergonic in our system, we did not search extensively for additional pathways for conversion of the aldehyde to ethanol. 


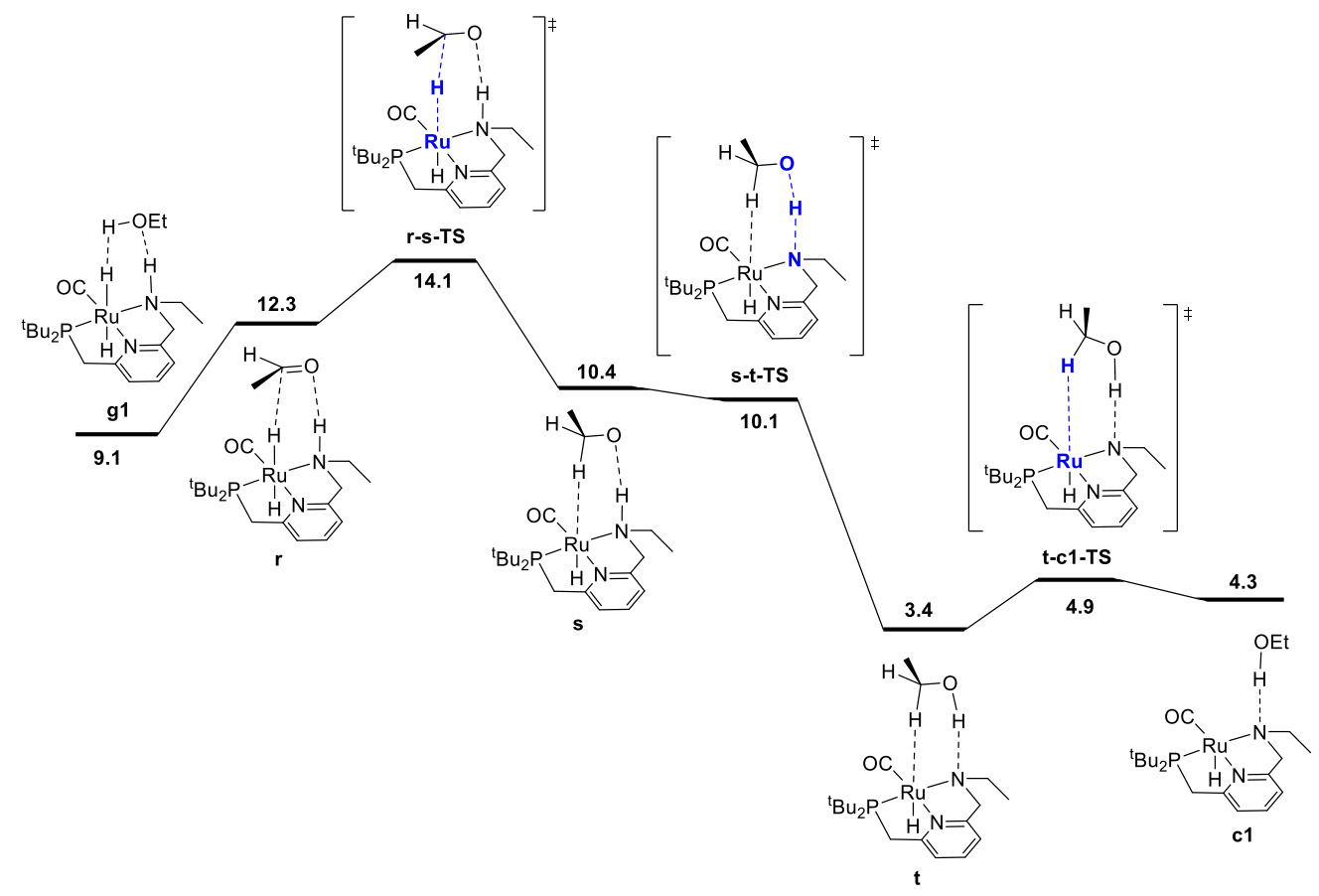

Figure 7. Minimum-energy pathway for hydrogenation of acetaldehyde to ethanol, accompanied by conversion of dihydride intermediate $\mathbf{g} \mathbf{1}$ into unsaturated intermediate $\mathbf{c 1}$, which connects back to the hydrogen-activation pathway shown in Figure 1. For consistency in this energy diagram, the free energy of intermediate $\mathbf{g} 1(9.1 \mathrm{kcal} / \mathrm{mol})$ is calculated based on the organic intermediates ethanol, acetaldehyde, and one molecule of hydrogen, whereas the free energy of $2.8 \mathrm{kcal} / \mathrm{mol}$ shown in Figures 1 and 4 is based on the organic reactants ethyl acetate and two molecules of hydrogen. This presentation ensures that free energy changes within each figure are correct (e.g. the standard-state free energy change on substituting ethanol for acetaldehyde in converting from $\mathbf{g} 1$ to $r$ is $3.2 \mathrm{kcal} / \mathrm{mol}$ as shown in this figure).

Summary and Predicted Kinetics. In summary, we have identified minimum-energy pathways for hydrogen activation, ester hydrogenolysis, and aldehyde hydrogenation in ester hydrogenation catalyzed by RuPNN ${ }^{\text {HEt }}$, which forms in situ from RuPNN ${ }^{\text {imine }}$ as we have shown experimentally. ${ }^{9}$ Figure 8 shows a simplified energy diagram depicting key intermediates and transition states relevant in predicting the kinetics of hydrogenation through the energetic span model. ${ }^{15}$ The hydrido-alkoxide complex $\mathbf{a} \mathbf{2}$ is predicted to be the turnover-frequency-determining intermediate (TDI). The highestenergy transition states in the hydrogen activation and ester hydrogenolysis sequences are f2-g2-TS and $\mathbf{m}-\mathbf{n}$-TS, respectively. Although intermediate $\mathbf{n}$ is calculated to be higher than $\mathbf{m}-\mathbf{n}-\mathbf{T S}$ by $0.3 \mathrm{kcal} / \mathrm{mol},{ }^{20}$ we have used $\mathbf{m}$-n-TS in our kinetic analysis for consistent application of transition-state theory to calculate rate constants. The $2.1 \mathrm{kcal} / \mathrm{mol}$ free-energy difference between $\mathbf{f 2 - g 2 - T S}$ and $\mathbf{m}$ - $\mathbf{n}$-TS is likely within the error of the DFT method, especially considering the changes in molecularity involved: between a2 and f2-g2-TS, a hydrogen molecule is consumed, and between $\mathbf{f 2 - g 2 - T S}$ and $\mathbf{m}-\mathbf{n}$-TS, two ethanol molecules are released and ethyl acetate is consumed. 


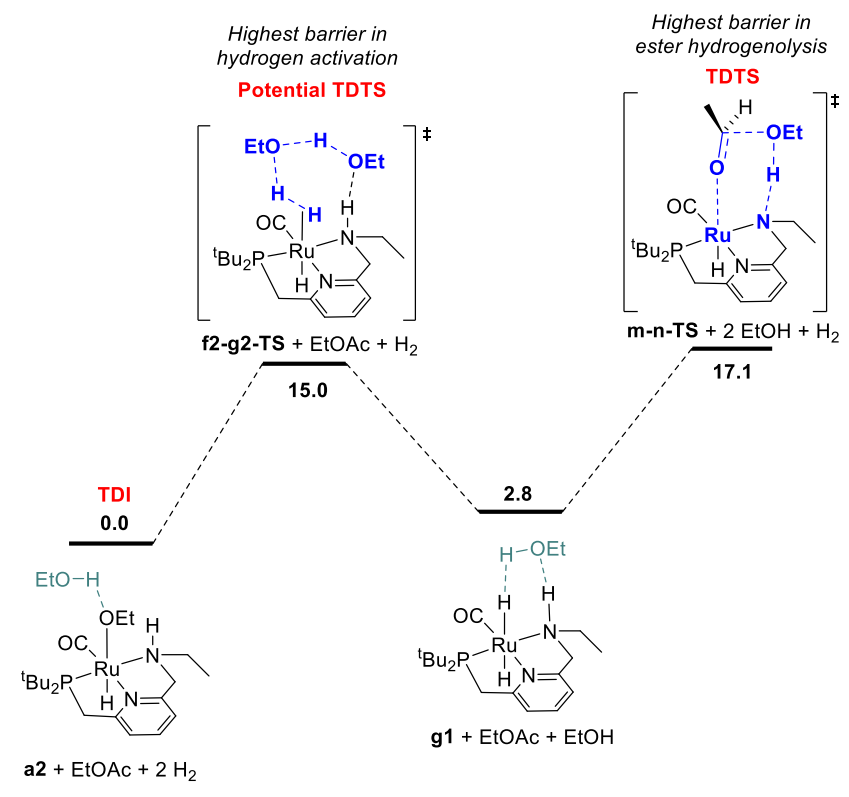

Figure 8. Simplified energy surface determining the kinetics of ester hydrogenation.

The energetic span model allows a prediction of the rate of catalysis based on the free-energy difference between the TDI and the TDTS, which is an effective barrier for catalytic turnover. ${ }^{15}$ Taking our DFT results at face value, the TDTS is $\mathbf{m}-\mathbf{n}$-TS when the reactants, EtOAc and $\mathrm{H}_{2}$, as well as the product $\mathrm{EtOH}$, are at their standard states of $1 \mathrm{M}$. In this scenario, the energetic span is $17.1 \mathrm{kcal} / \mathrm{mol}$, which is qualitatively consistent with a catalytic system that turns over rapidly at room temperature. The predicted rate law, based on the species consumed and released between the TDI and TDTS, is represented by Equation 1. In our experimental kinetic analysis described below, we have taken the above simplified model as a starting point, and additionally we find saturation behavior at high [ester], consistent with a switch to f2-g2-TS as TDTS under these conditions.

$$
\text { rate }=k \frac{[\mathrm{Ru}]\left[\mathrm{H}_{2}\right][\mathrm{EtOAc}]}{[\mathrm{EtOH}]^{2}}
$$

Effect of Explicit Ethanol on Kinetics. Motivated by past work showing the key involvement of protic solvent in heterolytic hydrogen cleavage ${ }^{23}$ and by the complicated dependence of our catalytic rate on alcohol concentration (as described below), we examined the effect of including explicit ethanol molecules in the hydrogen activation and ester hydrogenolysis pathways described above. The complete pathways are included in the Supporting Information. Figure 9 shows a summary of the effect of explicit ethanol molecules on the free energies of key intermediates and transition states that determine the kinetics. Taking the computed free energies at face value, several predictions can be made about the kinetics. First, the hydrido-alkoxide intermediate a2 interacts strongly with an ethanol molecule from solution, so the "free" complex a1 does not represent a significant fraction of the resting catalyst speciation, even at very low ethanol concentration. Second, the minimum-energy pathway for hydrogen activation goes through f2-g2-TS and includes two ethanol molecules as a "proton brigade", although a pathway through e1-g1-TS, with only one ethanol molecule as a proton shuttle, is only $3.6 \mathrm{kcal} / \mathrm{mol}$ higher. Third, as the energies of $\mathbf{g}$, g1, and $\mathbf{g} \mathbf{2}$ - the dihydride intermediates with 0,1 , and 2 ethanol 
molecules included - are above the energy of $\mathbf{a} \mathbf{2}$ and below the energies of the transition states, their specific energies and relative ordering are not kinetically relevant. Last, ester hydrogenolysis proceeds through a very similar free-energy barrier whether an explicit ethanol molecule is included (m1-n1-TS, $17.4 \mathrm{kcal} / \mathrm{mol}$ ) or not $(\mathrm{m}-\mathrm{n}-\mathrm{TS}, 17.1 \mathrm{kcal} / \mathrm{mol})$. This model formed the basis for our kinetic analysis, described below.

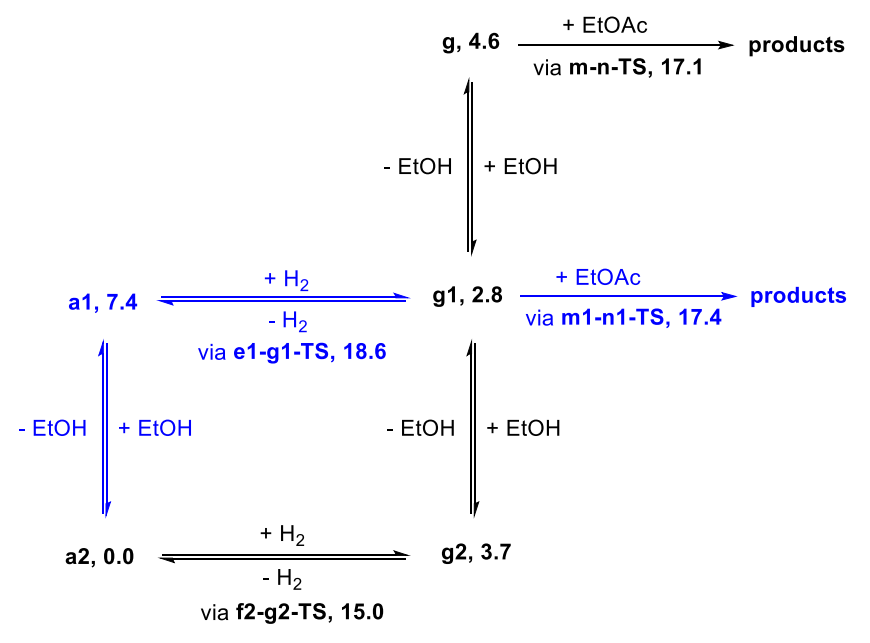

Figure 9. Simplified kinetic model of the MEP for ester hydrogenation (black), and reasonable alternative pathways including different numbers of explicit ethanol molecules (blue). Numbers given after each species represent standard-state free energies $(\mathrm{kcal} / \mathrm{mol})$ at $298.15 \mathrm{~K}$.

\section{Synthesis of Proposed Intermediates}

RuPNN $^{\text {HEt }}$. The computational studies described above predict that the catalyst resting state will be a hydrido-alkoxide species such as a2, stabilized by hydrogen-bonding to a product alcohol molecule. Dihydride species such as $\mathbf{g}, \mathbf{g} \mathbf{1}$, and $\mathbf{g} \mathbf{2}$ are predicted to be key intermediates, but are less stable by several $\mathrm{kcal} / \mathrm{mol}$ and are expected to have low steady-state concentrations once even small amounts of alcohol product build up in ester hydrogenation reactions. We previously demonstrated (Scheme 2 ) that the precatalyst RuPNN ${ }^{\text {imine }}$ converts quantitatively to the dihydride RuPNN ${ }^{\text {HEt }}$ under hydrogen pressure, ${ }^{9}$ although the reversibility of this reaction on removal of hydrogen prevented easy isolation of RuPNN ${ }^{\text {HEt }}$. Recently, Gusev reported a clever method to isolate the dihydride product RuPNN ${ }^{\mathrm{H2}}$ formed by reaction of Milstein's original precatalyst RuPNN ${ }^{\text {dearom }}$ with hydrogen: a solution of the dearomatized species was placed under hydrogen in an unstirred pressure vessel, in a solvent mixture that dissolved RuPNN ${ }^{\text {dearom }}$ completely but allowed the product dihydride to crystallize. ${ }^{13 c}$ Gratifyingly, we found that the same procedure allowed us to successfully isolate RuPNN ${ }^{\text {HEt }}$ in crystalline form (Scheme 4). RuPNN ${ }^{\text {HEt }}$ is isolated as yellow crystals, which are moderately stable at room temperature, but can be stored under argon at $-37^{\circ} \mathrm{C}$ for extended periods without decomposition. Although RuPNN ${ }^{\text {HEt }}$ is stable even under air as a solid, it decomposes rapidly when dissolved in degassed benzene- $d_{6}$ at room temperature. As we previously characterized RuPNN ${ }^{\text {HEt }}$ fully in solution under $\mathrm{H}_{2}$ pressure, ${ }^{9}$ we did not attempt to repeat the spectroscopic characterization in the absence of $\mathrm{H}_{2}$. 


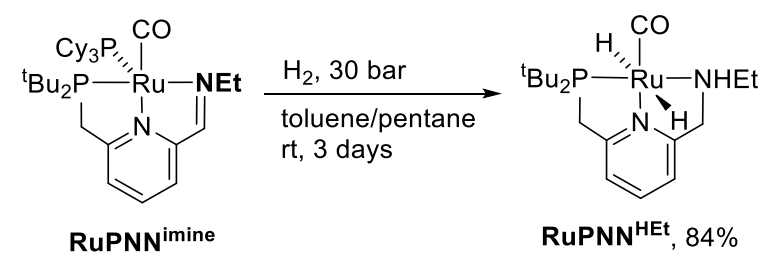

Scheme 4. Synthesis of RuPNN ${ }^{\text {HEt }}$.

The crystals of RuPNN ${ }^{\text {HEt }}$ formed by the method described above were suitable for characterization by X-ray crystallography. Figure 10 shows the molecular structure in the solid state. As was concluded based on our previous spectroscopic characterization, ${ }^{9}$ RuPNN ${ }^{\text {HEt }}$ features a nearly octahedral ruthenium(II) center bound to two hydride ligands, carbon monoxide, and a PNN-pincer ligand with an aromatic pyridine fragment flanked by $\mathrm{CH}_{2} \mathrm{P}\left({ }^{\mathrm{t}} \mathrm{Bu}\right)_{2}$ on one side and $\mathrm{CH}_{2} \mathrm{NHEt}$ on the other. The $\mathrm{NH}$ group is pseudo-equatorial and the NEt group is pseudo-axial. The structure is closely analogous to that of RuPNN $^{\mathrm{H2}}$ as recently reported by Gusev, ${ }^{13 \mathrm{C}}$ except for the change from $\mathrm{NEt}_{2}$ to NHEt. The mechanism of double hydrogenation from RuPNN ${ }^{\text {imine }}$ to give RuPNN ${ }^{\text {HEt }}$ is not obvious, and is the subject of an ongoing experimental and computational study.

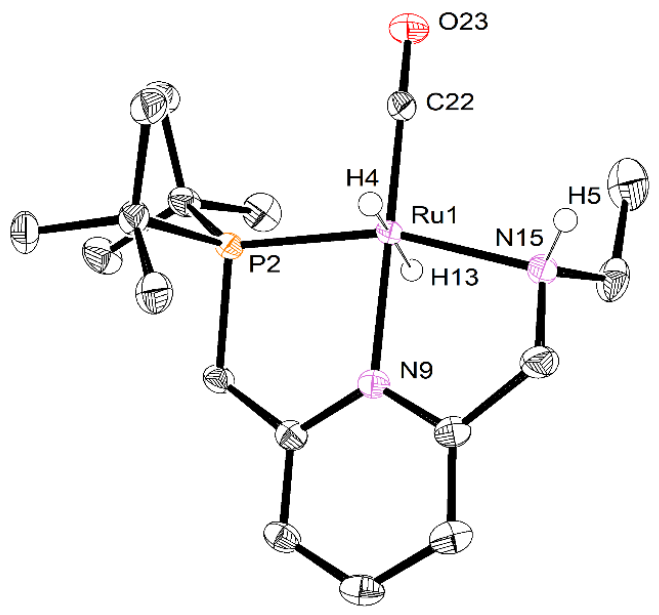

Figure 10. ORTEP diagram of RuPNN ${ }^{\mathrm{HEt}}$, showing $50 \%$ probability ellipsoids. Hydrogen atoms other than $\mathrm{Ru}-\mathrm{H}$ and $\mathrm{N}-\mathrm{H}$ are omitted for clarity. Selected bond lengths (Angstroms) and angles (degrees): $\mathrm{Ru}(1)-$ $\mathrm{P}(2), 2.2536(6) ; \mathrm{Ru}(1)-\mathrm{N}(15), 2.1893(19) ; \mathrm{Ru}(1)-\mathrm{N}(9), 2.0980(19) ; \mathrm{Ru}(1)-\mathrm{C}(22), 1.830(2) ; \mathrm{C}(22)-\mathrm{O}(23)$, 1.164(3); $P(2)-R u(1)-N(9), 82.45(6) ; N(9)-R u(1)-N(15), 78.71(7)$.

RuPNN ${ }^{\text {HOEt }}$. Although dihydride species such as RuPNN ${ }^{\mathrm{H} 2}$ have been proposed as the resting states in catalytic hydrogenation reactions, ${ }^{5 h}, 13 a, 24$ our calculations indicate that alkoxide a2 is more stable than the dihydride $\mathbf{g} 1$ by $2.9 \mathrm{kcal} / \mathrm{mol}$. In recent work, Gusev demonstrated experimentally that RuPNN ${ }^{\mathbf{H 2}}$ converts rapidly to alkoxide species on addition of alcohols, and showed computationally that the ethoxide species was more stable than the dihydride by $0.4 \mathrm{kcal} / \mathrm{mol} .{ }^{13 \mathrm{c}}$ We observed analogous reactivity for RuPNN ${ }^{\text {HEt}}$ : although it decomposes in benzene- $d_{6}$ with no other additives, RuPNN ${ }^{\text {HEt }}$ rapidly converts to the hydrido-alkoxide species RuPNN ${ }^{\text {HOEt }}$ when dissolved in benzene- $d_{6}$ containing ethanol, 
with visible evolution of hydrogen gas (Scheme 5). NMR spectra taken immediately after reaction show one clean species. RuPNN ${ }^{\text {HOEt }}$ was fully characterized by NMR spectroscopy at $25^{\circ} \mathrm{C}$ in benzene- $d_{6}$. The hydride signal appears as a doublet at $-15.8 \mathrm{ppm}$. At room temperature, broad signals are observed for the methylene and hydroxyl hydrogens of free ethanol. Signals for the bound ethoxide, $\mathrm{N}-\mathrm{H}$, and the $\mathrm{PCH}_{2}$ hydrogen syn to the ethoxide are not observed, as they are in rapid exchange with hydrogens from free ethanol. To characterize RuPNN ${ }^{\text {HOEt }}$ in the absence of this chemical exchange, ${ }^{1} \mathrm{H}$ NMR spectra were recorded from $-90^{\circ} \mathrm{C}$ to $20^{\circ} \mathrm{C}$ in toluene- $\mathrm{d}_{8}$ (see the Supporting Information for spectral images). At -50 ${ }^{\circ} \mathrm{C}$, the above chemical exchanges are slow on the NMR time scale, and distinct resonances are observed for free ethanol, bound ethoxide, the $\mathrm{N}-\mathrm{H}$, and all four $\mathrm{CH}_{2}$ linker hydrogens.

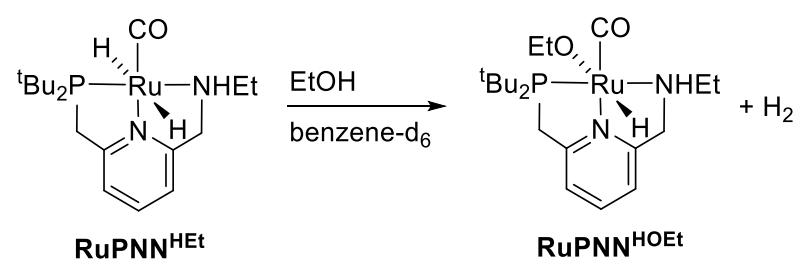

Scheme 5. Synthesis of RuPNN ${ }^{\text {HOEt }}$.

Single crystals of RuPNN ${ }^{\text {HOEt }}$ suitable for X-ray crystallography were obtained by slow evaporation of a pentane solution containing a small amount of ethanol. Although crystals could be reproducibly obtained in this manner, the instability of RuPNN ${ }^{\text {HOEt }}$ in the absence of an excess of ethanol coupled with its high solubility in solvents with a wide range of polarities have thus far prevented its bulk isolation as a solid. Figure 11 shows the structure of RuPNN ${ }^{\text {HOEt }}$. In the solid state, the ethoxide ligand is syn to the $\mathrm{N}-\mathrm{H}$ group, which is pseudo-axial and is $2.21 \AA$ from the ethoxide oxygen, indicating a weak intramolecular hydrogen bond. A molecule of ethanol is present in the asymmetric unit, and the $\mathrm{O}-\mathrm{H}$ hydrogen interacts with the ethoxide oxygen through hydrogen-bonding with a distance of $1.73 \AA$. The solid-state geometric parameters for RuPNN ${ }^{\text {HOEt }}$ are remarkably similar to the computationally optimized structure $\mathbf{a 2}$, which was the lowest-energy hydrido-alkoxide conformation we were able to locate that included one explicit ethanol molecule. 


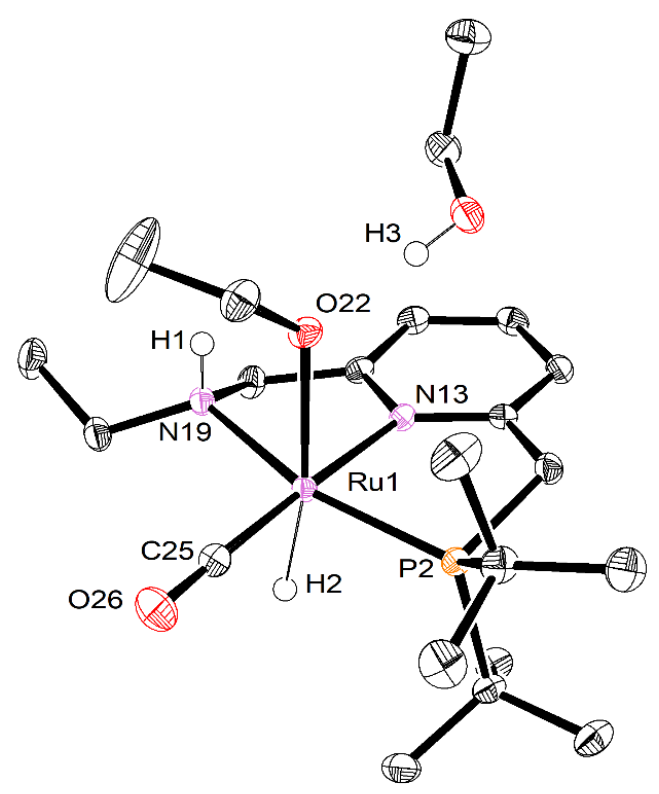

Figure 11. ORTEP diagram of RuPNN ${ }^{\text {HOEt }}$, showing $50 \%$ probability ellipsoids. Hydrogen atoms other than $\mathrm{Ru}-\mathrm{H}, \mathrm{N}-\mathrm{H}$, and $\mathrm{O}-\mathrm{H}$ are omitted for clarity. Selected bond lengths (Angstroms) and angles (degrees): $\mathrm{Ru}(1)-\mathrm{P}(2), 2.2671(5) ; \mathrm{Ru}(1)-\mathrm{N}(13), 2.1056(14) ; \mathrm{Ru}(1)-\mathrm{N}(19), 2.1693(14) ; \mathrm{Ru}(1)-\mathrm{O}(22), 2.1980(12)$; $\mathrm{Ru}(1)-\mathrm{C}(25), 1.8357(19) ; \mathrm{C}(25)-\mathrm{O}(26), 1.159(2) ; \mathrm{H}(1)-\mathrm{O}(22), 1.725 ; \mathrm{H}(3)-\mathrm{O}(22), 2.212 ; \mathrm{P}(2)-\mathrm{Ru}(1)-\mathrm{N}(13)$, $82.60(4) ; N(13)-R u(1)-N(19), 77.78(5)$.

The rapid conversion of RuPNN ${ }^{\text {HEt }}$ to RuPNN ${ }^{\text {HOEt }}$ at room temperature is consistent with our DFT study above: this transformation is the reverse reaction of the hydrogen activation shown in Figure 1 which is predicted to proceed in the reverse direction with a free-energy barrier of $12.1 \mathrm{kcal} / \mathrm{mol}$, proceeding from g1 through f2-g2-TS. The complete formation of RuPNN ${ }^{\text {HOEt }}$ from RuPNN ${ }^{\text {HEt }}$ suggested that, as predicted by computation, the alkoxide species might be more stable under catalytic conditions, and would hence represent the resting state and TDI. To confirm this, we placed a solution of RuPNN ${ }^{\text {HOEt }}$ formed in situ from RuPNN ${ }^{\text {HEt }}$ and ethanol under 10 bar $\mathrm{H}_{2}$ in a high-pressure NMR tube. No conversion back to the dihydride species was observed, consistent with the prediction from computation that RuPNN ${ }^{\text {HOEt }}$ is the dominant resting state throughout the catalytic reaction.

\section{Kinetics}

As we noted in the introduction, DFT studies of catalytic ester hydrogenation are widespread but kinetic studies are rare. Because the computed mechanism and energies make clear predictions about the kinetics, the latter provide an important check on the former. Based on our computed mechanism, the following predictions can be made. First, because hydrogen activation occurs between the TDI and TDTS, the reaction should be first-order in hydrogen. If a dihydride intermediate such as $\mathbf{g} 1$ were more stable than the intermediate preceding hydrogen activation ( $\mathbf{2} \mathbf{2}$ in our work), the reaction would follow zero-order kinetics in hydrogen. Second, because an ester molecule is consumed between the TDI and TDTS, the reaction should be first-order in ester. If the barrier for hydrogen activation were much higher than the barrier for ester hydrogenolysis, the reaction would follow zero-order kinetics in ester. If these 
two barriers are similar in energy, saturation behavior is possible. Third, because alcohol product is released between the TDI and TDTS, the reaction should be inhibited by the buildup of alcohol. The precise dependence of the rate on [alcohol] is not unambiguously predicted by computation, because of the multiple pathways available as shown in Figure 9 above. Lastly, and very importantly, the overall rate of reaction should be approximately consistent with the overall barrier predicted by DFT, which is $17.1 \mathrm{kcal} / \mathrm{mol}$ in our system.

Although the hydrogenation of ethyl acetate to ethanol was ideal for our computational study and for the isolation of the hydrido-alkoxide intermediate RuPNN ${ }^{\text {HOEt }}$, we chose to conduct detailed kinetic studies using hexyl hexanoate as substrate, because both the ester reactant and alcohol product have very low volatilities at room temperature, which minimizes the possibility of evaporation of reactant or product at any point during the setup, reaction, or analysis. As both RuPNN ${ }^{\text {HEt }}$ and RuPNN ${ }^{\text {HOEt }}$ were unstable in solution, we conducted kinetic studies using RuPNN ${ }^{\text {imine }}$ as precatalyst. We previously determined that isopropyl alcohol was an ideal solvent for obtaining high catalytic rates and turnover numbers in practical ester hydrogenation catalyzed by RuPNN ${ }^{\text {imine }}{ }^{9}$ but we decided to conduct kinetic studies in toluene for two reasons: 1 ) the RuPNN ${ }^{\text {imine }}$ precatalyst is only sparingly soluble in isopropyl alcohol, posing difficulties with the preparation of stock solutions and occasionally causing clogging in the stainless-steel tubing used for removing aliquots from the reaction mixture; and 2) as described below, the hexanol product was found to inhibit turnover, and the analysis of this observed product inhibition was most straightforward if no other alcohols were present in solution.

In kinetic experiments, we monitored the conversion of hexyl hexanoate to 1-hexanol at $25^{\circ} \mathrm{C}$ by gas chromatography, with tetradecane as an internal standard. We began with the standard conditions shown in Scheme 6, then varied the initial concentrations of RuPNN ${ }^{\text {imine }}$, hexyl hexanoate, and hexanol, as well as the hydrogen pressure in independent experiments. The plots labeled "without preactivation" in Figure 12 shows a typical kinetic trace under our standard conditions. Over approximately the first 45 minutes of the reaction, the rate increases, after which apparent pseudo-first-order consumption of ester is observed, as the plot of In[ester] vs time is linear after this point. During the 45-minute induction period, aliquots are dark purple, indicating the presence of the strongly absorbing precatalyst RuPNN ${ }^{\text {imine }}$, and become pale yellow as the precatalyst is converted to the resting state, which we propose is a hydrido-hexyloxide species analogous to RuPNN ${ }^{\text {HOEt }}$.

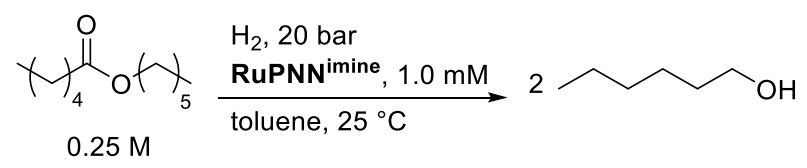

Scheme 6. Standard conditions for kinetic experiments. 

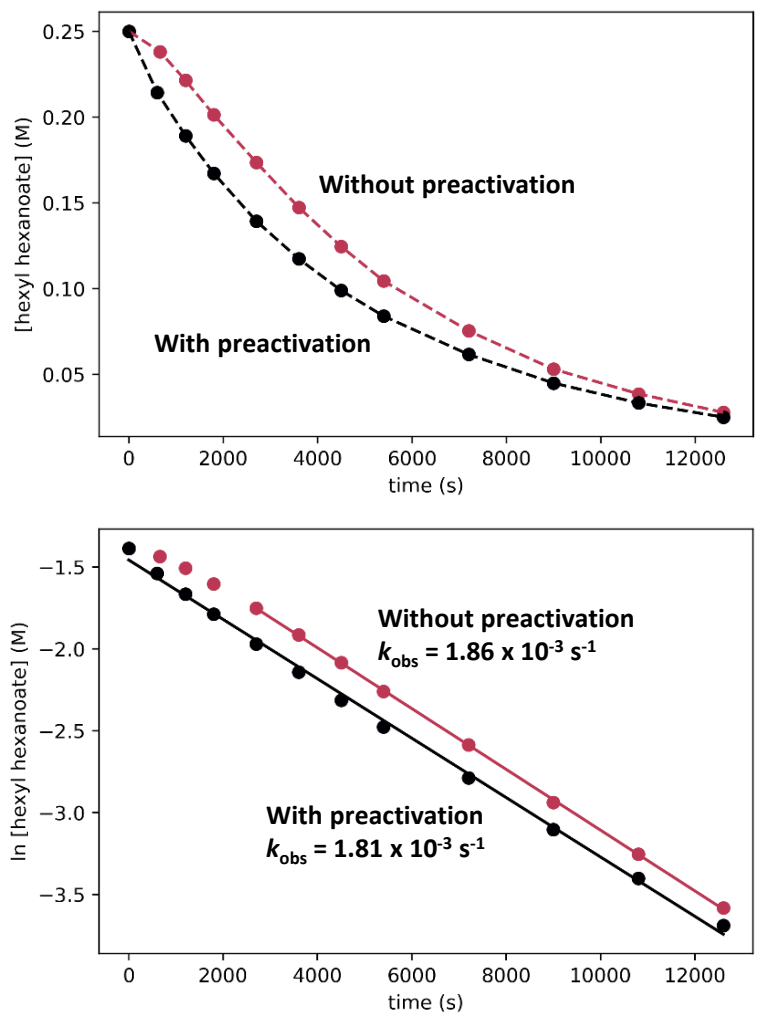

Figure 12. Comparison of hexyl hexanoate hydrogenation catalyzed by RuPNN ${ }^{\text {imine }}$ with and without preactivation of the catalyst by incubation under 20 bar hydrogen for 90 minutes. The top plots show [ester] vs. time; dashed lines are merely to guide the eye and do not represent a fit to the data. The bottom plots show $\ln$ [ester] vs. time, with linear fits to all data (with preactivation) or to the time points from 45 minutes on (without preactivation).

To determine if the observed induction period can be explained by the activation of RuPNN $\mathbf{N}^{\text {imine }}$ with hydrogen, we conducted a preactivation experiment where we first pressurized a solution of RuPNN ${ }^{\text {imine }}$ with hydrogen (20 bar) for 90 minutes, which results in formation of the dihydride complex RuPNN ${ }^{\text {HEt }}{ }^{9}$ Then, the hexyl hexanoate substrate was transferred into the pressure reactor and its conversion to 1-hexanol was monitored at $25^{\circ} \mathrm{C}$. As the plots labeled "with preactivation" in Figure 12 demonstrate, the reaction follows apparent first-order kinetics without an induction period, giving a nearly identical $k_{\text {obs }}$ value to what is observed without pre-activation of the catalyst. Importantly, this experiment rules out the possibility that sigmoidal kinetics come from acceleration of the reaction by the product alcohol, as proposed by $\mathrm{O}$ and Morris for their catalyst. ${ }^{5 a}$ We also attempted to use RuPNN $^{\text {HEt }}$ directly as a precatalyst, but partial decomposition prior to the introduction of hydrogen pressure hindered our attempts to obtain reliable kinetic data. Because it was much more operationally convenient to assemble kinetic experiments in parallel without a catalyst preactivation step, we elected to conduct further kinetic trials using RuPNN ${ }^{\text {imine }}$ as the precatalyst, using only the data after the 45minute induction period to develop the kinetic model for the activated catalyst.

To determine the partial order in catalyst concentration under the standard conditions, we repeated the experiment with a range of initial concentrations of RuPNN ${ }^{i m i n e}$ (Figure 13). The same initial 
induction period followed by pseudo-first-order behavior was observed, and $k_{\text {obs }}$ was taken as the slope of the linear portion of the plot of In[ester] vs time. A plot of $k_{\text {obs }}$ Vs. [RuPNN $\left.{ }^{\text {imine }}\right]_{0}$ is linear with an intercept of zero, indicating that the reaction is first-order in [ruthenium] after the induction period is complete.
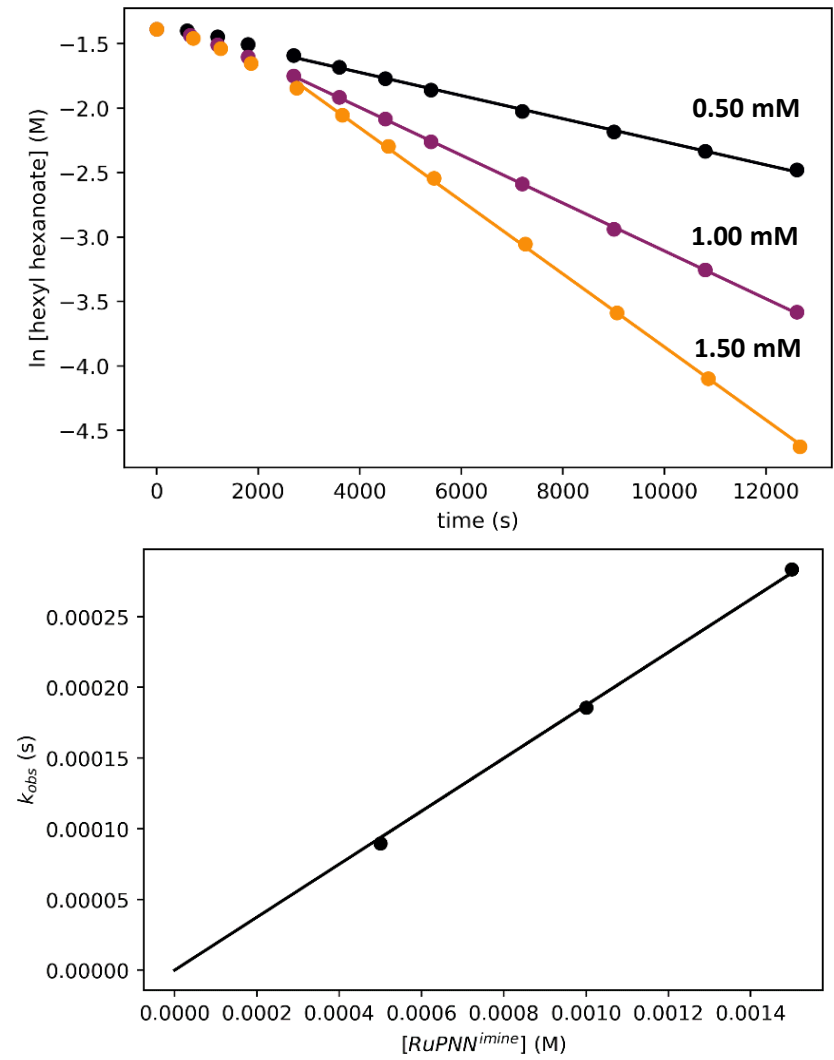

Figure 13. Determination of the partial order in RuPNN ${ }^{\text {imine }}$ under the standard conditions. The top plots show the time course of ester conversion using different initial concentrations of RuPNN ${ }^{\text {imine }}$, along with linear fits to the logarithm of [ester], using data after the induction period of 45 minutes. The bottom plot shows the linear relationship between $\mathrm{k}_{\mathrm{obs}}$ and [RuPNN ${ }^{\text {imine}}$ ].

To determine the partial order in hydrogen, we repeated the standard experiment varying the hydrogen pressure (Figure 14). In all experiments, a constant hydrogen pressure was maintained as aliquots were removed. Again, $k_{\text {obs }}$ was determined based on the linear portion of the plot of In[ester] vs. time, and again a plot of $k_{\mathrm{obs}}$ vs. hydrogen pressure gave a line with an intercept of zero, indicating that the reaction is first-order in hydrogen under these conditions. 

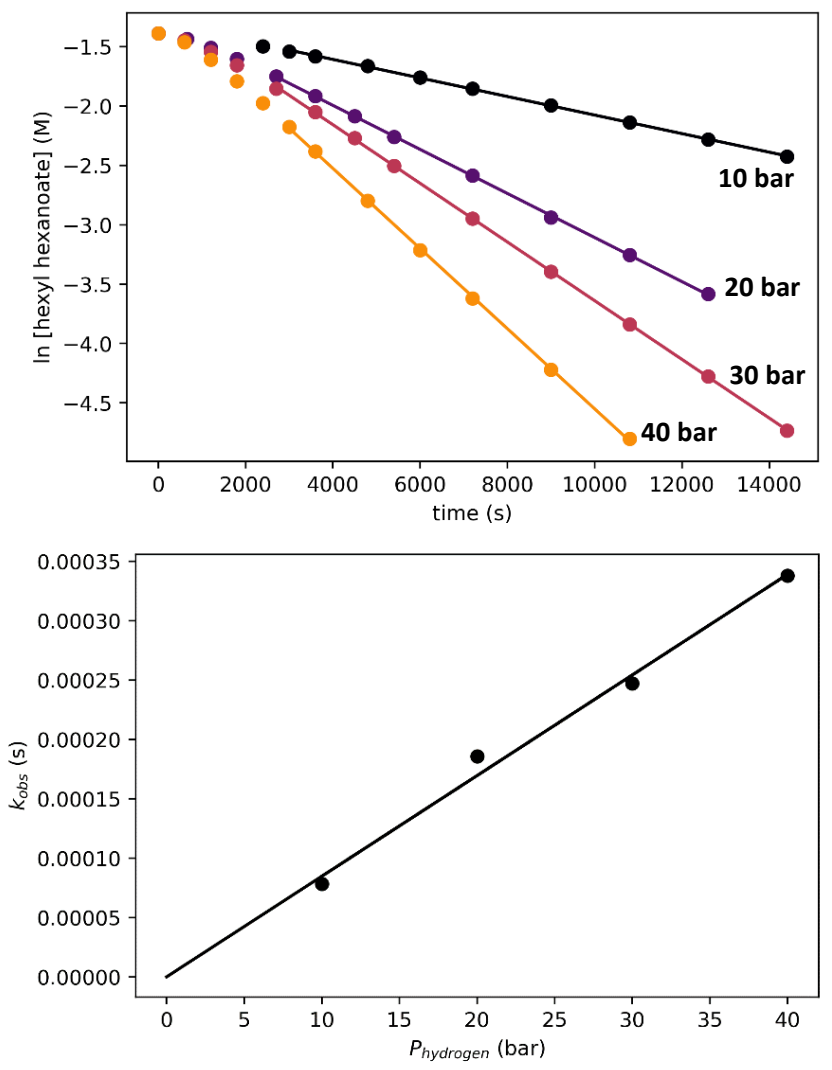

Figure 14. Determination of the partial order in hydrogen under the standard conditions. The top plots show the time course of ester conversion, along with linear fits to the logarithm of [ester], using data after the induction period of 45 minutes. The bottom plot shows the linear relationship between $\mathrm{k}_{\mathrm{obs}}$ and hydrogen pressure.

We then repeated the experiment with initial ester (hexyl hexanoate) concentrations varied over a wide range from $0.05 \mathrm{M}$ to $0.75 \mathrm{M}$. At high [ester], we observed a change from pseudo-first-order to pseudo-zero-order behavior, consistent with saturation kinetics. When [ester $]_{0}$ is $0.25 \mathrm{M}$ or less, apparent first-order kinetic behavior is observed in each individual experiment, but $k_{\text {obs }}$ increases dramatically at lower [ester] $]_{0}$ consistent with inhibition by the product 1-hexanol. Although it may be counterintuitive that pseudo-first-order kinetic behavior in [ester] is observed when the buildup of alcohol product inhibits the reaction, in our system the increasing product inhibition is roughly cancelled out by saturation in [ester], resulting in apparent first-order behavior in each experiment. To directly probe for inhibition by the product hexanol, we repeated the experiment with a range of initial 1hexanol concentrations, and we found that the rate qualitatively decreases as [hexanol] $]_{0}$ is increased, consistent with product inhibition.

To deconvolute the effects of saturation in [ester] and inhibition by the product alcohol, we developed a numerical model of the reaction progress using the program Copasi. ${ }^{25}$ Numerical modeling is seeing increased use in the analysis of kinetics in catalytic systems. ${ }^{16 b, 26}$ When used in combination with DFT, kinetic modeling offers the potential to validate mechanisms and refine the energies predicted by DFT. ${ }^{16 b}$, $26 \mathrm{e}$ In developing our model, we included the data after the 45-minute induction period from a 
total of 18 kinetic experiments, where the initial ester, alcohol, and ruthenium concentrations were varied, as well as the hydrogen pressure. Our model takes the standard-state free energies of the kinetically relevant intermediates and transition states as inputs (see Figure 9 above), and computes the time course for ester hydrogenation, given the initial concentrations of ruthenium catalyst (a2), hexyl hexanoate, hexanol, and hydrogen. Hydrogen concentration, calculated from its known solubility in toluene at $25^{\circ} \mathrm{C}$ and the appropriate pressure, ${ }^{27}$ was held fixed in the model. Because the activity coefficients of alcohols are known to vary significantly over the range of 0 to $1.0 \mathrm{M}$ in non-polar solvents, ${ }^{28}$ we used the activity of 1 -hexanol rather than its molarity, as estimated following a model developed by Li and coworkers for 1-hexanol in benzene. ${ }^{29}$

In attempting to reproduce the kinetic data with our model, we set the relative free energy of a2 to zero and compared a range of scenarios adjusting the remaining energies, in an attempt to achieve the best overall fit while including the smallest number of adjustable parameters. We found no better fit by allowing the free energies of a1, e1-g1-TS, g2, g1, or $\mathbf{g}$ to be adjusted. On the other hand, allowing adjustment of f2-g2-TS, $\mathbf{m - n - T S}$, and $\mathbf{m 1 - n 1 - T S}$ was essential to obtaining a good global fit. Further, entirely excluding the pathway through e1-g1-TS had no detrimental effect on the fit. Our optimized model is depicted in Figure 15, and the global fit to the kinetic data is shown in Figure 16. The free energies of the dihydride species $\mathbf{g}$, g1, and $\mathbf{g} \mathbf{2}$ were taken from the DFT results and held constant. The free energies of the transition states $\mathbf{f 2 - g 2 - T S , ~} \mathbf{m}-\mathbf{n}$-TS, and $\mathbf{m 1 - n 1 - T S}$ were allowed to vary; fitted values are shown in Figure 15.

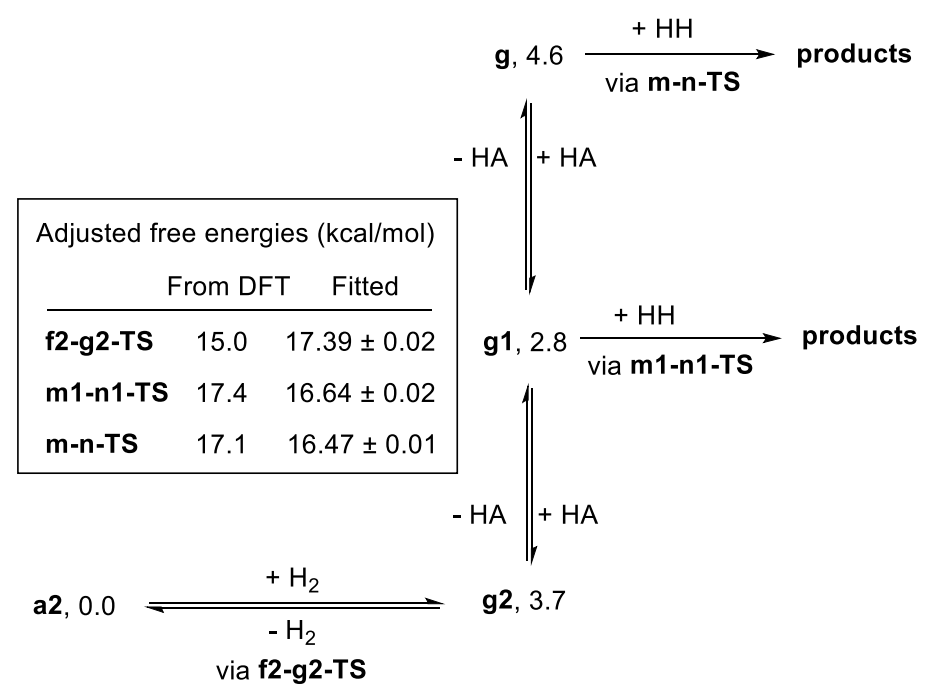

Figure 15. Optimized kinetic model. The free energies of a2, g2, g1, and $\mathbf{g}$ were held fixed at the indicated values. The free energies of f2-g2-TS, $\mathbf{m - n - T S}$, and $\mathbf{m 1 - n 1 - T S}$ were adjusted to achieve the best global fit to the kinetic data. HH refers to hexyl hexanoate and HA refers to 1-hexanol. 

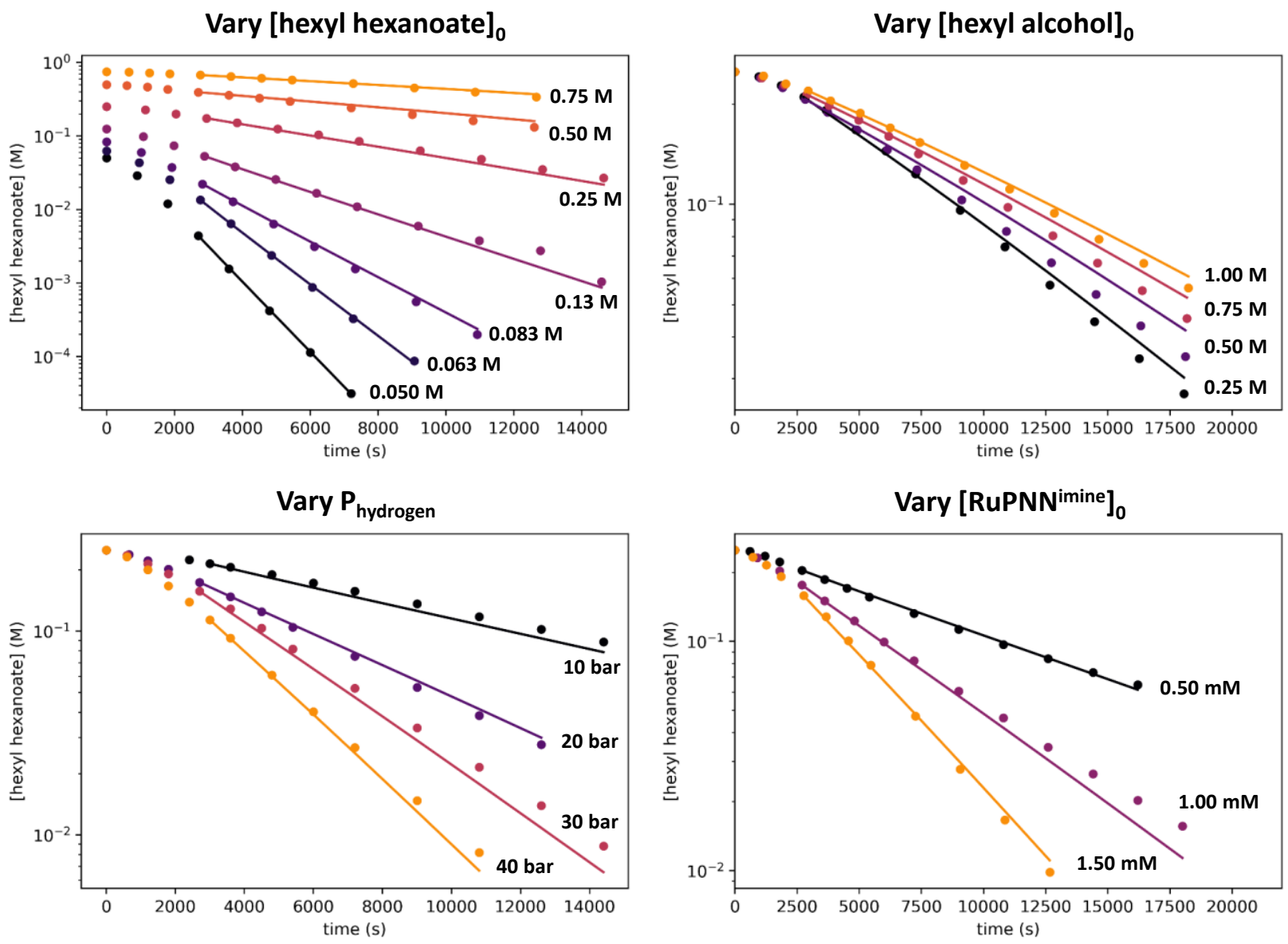

Figure 16. Data (points) and global fit (lines) for all 18 kinetic experiments. In the standard conditions, $[\text { hexyl hexanoate }]_{0}=0.25 \mathrm{M}$, [hexyl alcohol $]_{0}=0 \mathrm{M},\left[\text { RuPNN }^{\text {imine }}\right]_{0}=1.00 \mathrm{mM}$, and $P_{\text {hydrogen }}=20$ bar. The global fit was based on all data from 45 minutes on in each kinetic experiment. Note that the vertical axes are plotted logarithmically.

Overall, the kinetic data are very well-reproduced by this simplified model, with minimal adjustment of the free energies obtained from DFT. Interestingly, the free energy of the hydrogen activation transition state $\mathbf{f 2 - g 2 - T S}$ was adjusted upward by $2.4 \mathrm{kcal} / \mathrm{mol}$, while the energies of the ester hydrogenolysis transition states were adjusted downward by 1.2 and $1.4 \mathrm{kcal} / \mathrm{mol}$, indicating that the standard-state activation barrier for hydrogen cleavage is the higher of the two, in contrast to the prediction from DFT. The model correctly reproduces the first-order dependence on hydrogen pressure, the first-order dependence on [Ru], and the saturation kinetics in [ester]. The inclusion of two similarbarrier pathways for ester hydrogenolysis allows a transition from second-order inhibition by alcohol at low [alcohol] to first-order inhibition at higher [alcohol], consistent with the data. Importantly, the relatively small adjustment of the transition-state energies, less than $3 \mathrm{kcal} / \mathrm{mol}$ in each case, indicates that the barriers from DFT calculations are consistent with overall rate of the catalytic reaction. The above model satisfactorily reproduces the effect of [hexanol] on the rate of reaction essentially by assuming strong hydrogen bonding to the resting state RuPNN ${ }^{\text {HOEt }}$ and weaker interaction with the transition states for ester hydrogenation. However, we do not claim that this model fully accounts for 
the behavior of the alcohol in the system, which likely includes medium polarity effects and more complicated interactions with the reacting species.

Effect of Added Isopropyl Alcohol. Although we have conducted the above kinetic experiments in toluene, we determined previously ${ }^{9}$ that isopropyl alcohol was an ideal solvent for the reaction, giving rates approximately 2-3 times faster than toluene and THF. To probe the accelerating effect of isopropyl alcohol further, we repeated our standard kinetic experiment with varying amounts of isopropanol added, up to $0.75 \mathrm{M}$. For comparison, pure isopropyl alcohol is $13.1 \mathrm{M}$. As shown in Figure 17, we observe moderate but clear acceleration of the reaction with added isopropyl alcohol, consistent with our prior findings and in contrast with the inhibiting effect of added 1-hexanol. Although we have not tried to probe this effect further, it may originate from a different balance of stabilization of the resting state and transition-states by the two alcohols, potentially through specific interactions and/or medium polarity effects. For example, the activity coefficient of 1-hexanol is expected to decrease with increasing [isopropyl alcohol], which should reduce 1-hexanol inhibition and accelerate the catalytic reaction. We note that the detailed rate dependence determined above for hexyl hexanoate, especially the effect of the alcohol, does not necessarily extend to the hydrogenation of all other esters.
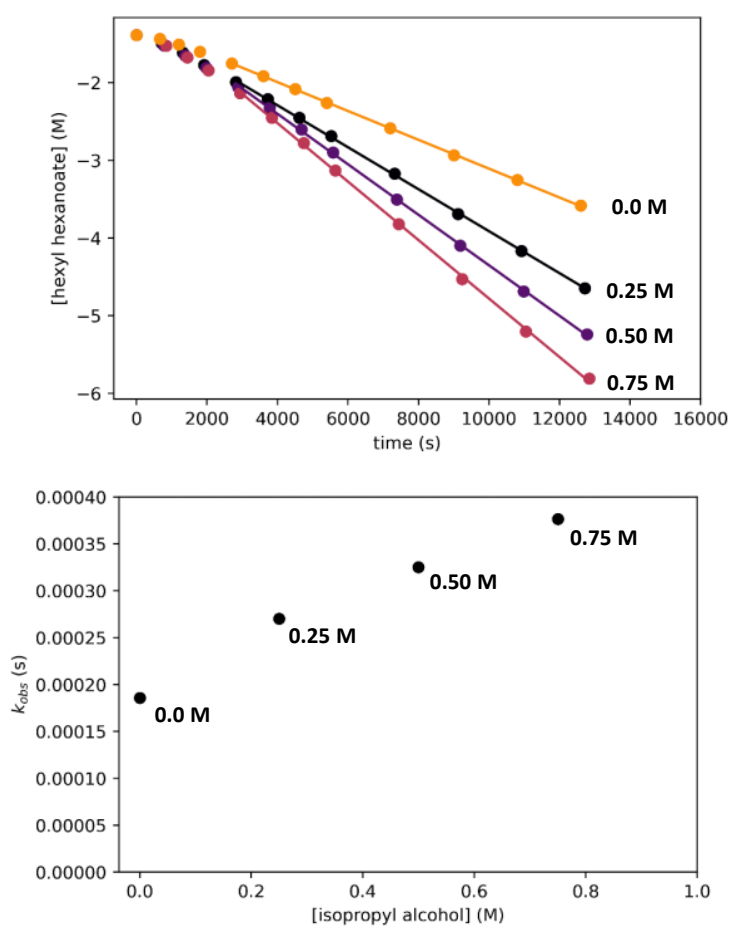

Figure 17. Effect of added isopropyl alcohol on the hydrogenation of hexyl hexanoate. The top plots show the time course of ester conversion using different initial concentrations of isopropyl alcohol, along with linear fits to the logarithm of [ester], using data after the induction period of 45 minutes. The bottom plot shows $k_{o b s} v s$. [isopropyl alcohol]. 
Disproportionation of Aldehydes to Esters. As Gusev has reported recently, ${ }^{30}$ catalysts for ester hydrogenation that produce an aldehyde intermediate can also be active for the catalytic disproportionation of aldehydes to esters, which effectively operates by running the ester hydrogenolysis pathway in reverse and the aldehyde hydrogenation pathway in the forward direction. Rearranging the pathways in Figures 4 and 7 gives the MEP shown in Figure S12 in the Supporting Information, with an overall barrier of $12.9 \mathrm{kcal} / \mathrm{mol}$. Therefore, our calculations predict that aldehyde disproportionation should be rapid at room temperature. To test this prediction, we dissolved 1-hexanal in benzene- $d_{6}$ with 0.2 mol \% RuPNN $^{\text {HEt }}$ at room temperature, and monitored by ${ }^{1} \mathrm{H}$ NMR. After 10 minutes, the aldehyde was completely consumed and hexyl hexanoate was the major product. (Scheme 7). Further studies of this disproportionation reaction are in progress.

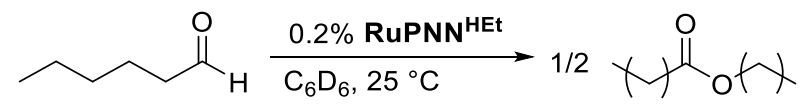

Scheme 7. Disproportionation of 1-hexanal.

\section{Discussion}

We previously demonstrated that the ubiquitous RuPNN ${ }^{\text {dearom }}$ is not kinetically competent as a catalyst for ester hydrogenation, instead converting from an inactive precatalytic form with an $\mathrm{NEt}_{2}$ side group to an active form RuPNN ${ }^{\text {HEt }}$, which features an NHEt group that is essential for catalytic activity. ${ }^{9}$ In this work, we have presented a plausible minimum-energy pathway, identified through computation and validated experimentally through kinetic characterization and isolation of two key intermediates, RuPNN $^{\text {HEt }}$ and RuPNN ${ }^{\text {HOEt }}$. Our computations demonstrate that the $\mathrm{N}-\mathrm{H}$ functional group plays a key role in the exceptional room-temperature activity of this catalyst. The $\mathrm{N}-\mathrm{H}$ group is deprotonated and reprotonated in our MEPs for hydrogen activation, ester hydrogenolysis, and aldehyde hydrogenation, although in the first two cases we identified nearly isoenergetic pathways where the $\mathrm{N}-\mathrm{H}$ group acts only as a hydrogen-bond donor without being deprotonated. A thorough search for alternative pathways where a $\mathrm{CH}_{2}$ linker is involved in hydrogen activation identified a minimum barrier of $23.7 \mathrm{kcal} / \mathrm{mol}$, compared to 15.0 in our MEP.

Because of the widespread application of RuPNN ${ }^{\text {dearom }}$ in catalytic transformations and the corresponding widespread study of its reactivity by DFT prior to our disclosure of its facile dehydroalkylative activation, this system provides a unique case study in how the application of DFT in the absence of complementary experimental data can lead to the proposal of incorrect reaction mechanisms. Three studies we are aware of report a complete pathway for ester hydrogenation catalyzed by RuPNN ${ }^{\text {dearom }}$. In 2017, Zhang and coworkers reported a mechanism for the hydrogenation of ethyl benzoate catalyzed by RuPNN ${ }^{\text {dearom }}{ }^{13 b}$ In their work, RuPNN ${ }^{\text {dearom }}$ was identified as the resting state, and the highest barrier occurred in ester hydrogenolysis, giving an energetic span of 27.2 $\mathrm{kcal} / \mathrm{mol}$. In 2011, Wang and coworkers reported a study comparing the activity of RuPNN ${ }^{\text {dearom }}$ for the acceptorless dehydrogenative coupling (ADC) of alcohols to give esters against the coupling of amines and alcohols to give amides, rationalizing the preference for the latter pathway over the former. ${ }^{13 a}$ Reversing the ADC process predicts an overall energetic span of $38.5 \mathrm{kcal} / \mathrm{mol}$ for ester hydrogenation, 
from a dihydride resting state after hydrogen activation to a proton-transfer TDTS along the ester hydrogenolysis pathway. In 2020, Gusev reported a revised mechanism for ester hydrogenation and the reverse $A D C$, aided by the experimental identification of a hydridoalkoxide species as the proposed resting state. ${ }^{13 c}$ In that study, the energetic span from the hydridoalkoxide TDI to the TDTS, a Hasanaynlike ${ }^{21}$ hydride-alkoxide metathesis transition state, was $31.8 \mathrm{kcal} / \mathrm{mol}$.

As all three of the above studies rely on the on-cycle intermediacy of either RuPNN ${ }^{\text {dearom }}$, RuPNN $\mathbf{N}^{\mathbf{H 2}}$, or both, the proposed mechanisms cannot be correct, as we have shown that RuPNN ${ }^{\text {dearom }}$, which converts rapidly to RuPNN ${ }^{\mathrm{H} 2}$ under hydrogen pressure, ${ }^{1}$ is inactive in ester hydrogenation prior to undergoing dehydroalkylation. ${ }^{9}$ With our present demonstration that the experimental free-energy barrier to catalytic turnover is only $17.4 \mathrm{kcal} / \mathrm{mol}$, the computed pathways above can also be excluded because the barriers they predict are implausibly high. Although it is not always explicitly stated, a common filter for the plausibility of reaction mechanisms calculated by DFT or other quantum-chemistry methods is a qualitative agreement of the overall reaction barrier with the observed rate of reaction. When detailed kinetic information is not available, reaction barriers must be estimated knowing only the catalyst loading, reaction time, and temperature. In the case of ester hydrogenation catalyzed by RuPNN ${ }^{\text {dearom }}$, Milstein's initial disclosure reported a turnover number of 100 in $4 \mathrm{~h}$ at $115^{\circ} \mathrm{C}^{1}{ }^{1}$ If one assumes that catalyst induction is rapid and the turnover frequency is constant over the reaction time course, an overall barrier (energetic span) of $26.7 \mathrm{kcal} / \mathrm{mol}$ can be estimated. However, the barrier for turnover can be substantially overestimated if the catalyst undergoes a slow activation followed by very rapid turnover, as we showed is the case for this system. ${ }^{9}$ This overestimation makes the above mechanisms, especially those proposed by Gusev and Zhang, appear plausible even though they predict barriers that are much higher than the actual barrier for catalytic turnover.

It is worth revisiting a broader implication of the findings we report here. As we described in the introduction, the majority of elite catalysts for ester hydrogenation and the reverse ADC of alcohols feature an $\mathrm{N}-\mathrm{H}$ group with a key role in promoting catalysis. In this work and in a prior study, ${ }^{9}$ we demonstrated that Milstein's catalyst RuPNN ${ }^{\text {dearom }}$ and NEt ${ }_{2}$-substituted CNN-pincer analogs developed in our group ${ }^{7 d}$ are initially inactive, and must convert to an NHEt form to be catalytically active. Recently, Khaskin, Gusev, and coworkers ${ }^{22}$ have shown that the same is true for a related bipyridyl PNN-pincer catalyst originally reported by Milstein and coworkers. ${ }^{31}$ In this case, a pyridine ring is hydrogenated to a piperidine, again providing a latent $\mathrm{N}-\mathrm{H}$ functional group with a key role in catalysis. Before these reports, initial proposals ${ }^{1-2,31}$ and many computational studies ${ }^{13 a, 14}$ pointed to the reversible deprotonation of a $\mathrm{CH}_{2}$ linker as a key step in catalysis, but these proposals should potentially be reevaluated in light of the new findings. Importantly, our work does not completely rule out the potential involvement of $\mathrm{CH}_{2}$ linkers in other processes. It is experimentally known that the addition of $\mathrm{H}_{2}$ to RuPNN ${ }^{\text {dearom }}$ occurs reversibly at room temperature (Scheme 1 ). ${ }^{1}$ Our calculations (Figure S5) indicate that this process has a barrier of $23.7 \mathrm{kcal} / \mathrm{mol}$ under the conditions of ester hydrogenation, which is too high to account for the fast room-temperature turnover the catalyst exhibits in this process, but could be accessible at a higher temperature in a different process. We are continuing to probe these possibilities in computational and experimental studies of related catalytic transformations.

\section{Conclusion}

We previously demonstrated that Milstein's pincer-ruthenium catalyst for ester hydrogenation and related reactions is activated by dehydroalkylation to give the active form, which contains an $\mathrm{NH}$ 
functional group that is essential for catalysis. ${ }^{9}$ In this work, we have presented a detailed computational and experimental study of the mechanism of ester hydrogenation catalyzed by this activated form, and conclude that participation of the N-H functional group is key in hydrogen activation, ester hydrogenolysis, and aldehyde hydrogenation. The catalyst speciation, the overall rate of reaction, and the dependence of the rate on the concentrations of reactants and products determined by experiment are in agreement with the mechanism predicted by DFT.

\section{Experimental Section}

Computational Methods. Density functional theory calculations were performed using the Gaussian 16 computational chemistry package, Revision B.01. ${ }^{32}$ The geometries and energies of all species were calculated using the hybrid functional B3LYP, ${ }^{33}$ augmented with the addition of empirical dispersion with Grimme's D3 dispersion corrections ${ }^{33}$ (referred to as B3LYP-D3). Ru was modeled with the effective core potential of Hay and $\mathrm{Wadt}^{34}$ and the accompanying uncontracted basis set (including $f$ polarization functions $)^{35}$ collectively known as LANL08(f)..$^{36}$ All other elements were modeled with the 6$311 \mathrm{G}(\mathrm{d}, \mathrm{p})$ basis set. ${ }^{37} \mathrm{~A}$ superfine integration grid was used for all calculations, which aided convergence of structures with loosely bound fragments such as explicit ethanol molecules. Complete structures with no truncations were used in all cases, and were optimized in solvent, using a polarizable continuum with radii and non-electrostatic terms from Truhlar and coworkers' SMD solvation model, and with dielectric constants chosen for toluene. ${ }^{38}$ Although geometry optimization in solvent is important to identify ionpair intermediates that might be missed in the gas phase, ${ }^{6 b}$ some intermediates (especially $\mathrm{H}_{2}$ and $\mathrm{C}-\mathrm{H}$ $\sigma$-complexes) and two transition states failed to converge in solvent. Geometry optimizations and frequency calculations used for free-energy corrections for j, eP, eP-eq, eN1-eq, eN1, eN1-g1-TS, eN1s, gP2-eq, gP2, and i1-p1-TS were done in the gas phase. Frequency calculations ensured the absence of imaginary vibrational modes in intermediates and the presence of exactly one imaginary mode in transition states. Intrinsic reaction coordinate calculations were employed to verify that transition states led to the specified minima. For the transition states a2-b2-TS, c2-d2-TS, i-j-TS, m-n-TS, s-t-TS, hd-id-TS, id-jd-TS, and od-pd-TS, one or both connected intermediates were calculated to be higher in free energy than the transition state, an outcome which is unusual but well-precedented. ${ }^{39}$ In each case, the energy of the intermediate was lower than the transition state on the solvent-corrected B3LYP-D3 electronic energy surface employed for geometry optimization. Standard state corrections were added in order to adjust from $1 \mathrm{~atm}$ to $1 \mathrm{M}$ for solution-phase free energies, amounting to $1.89 \mathrm{kcal} / \mathrm{mol}$ added to the free energy of each isolated molecule at $298.15 \mathrm{~K} .{ }^{40}$ Although the standard state for molecular hydrogen is sometimes taken as the gas at $1 \mathrm{~atm}$, we have used a $1 \mathrm{M}$ standard state in toluene, for consistency in computing reaction kinetics from the calculated free energies. The solvation-corrected electronic energies were further refined using the M06 functional, ${ }^{41}$ using the same LANL08(f) basis set for ruthenium and $6-311+G(2 d, 2 p)$ for all other atoms. All energies reported in the paper are standard-state free energies at $298.15 \mathrm{~K}$. A table of energies is provided in the Supporting Information, and geometries in Cartesian coordinates are included in a separate, compiled .XYZ file.

Synthesis of RuPNN ${ }^{\mathrm{HEt}}$. RuPNN ${ }^{\mathrm{HEt}}$ was previously characterized by NMR spectroscopy in solution under 10 bar $\mathrm{H}_{2}$ in $\mathrm{C}_{6} \mathrm{D}_{6}{ }^{9}$ Here we report the isolation of RuPNN ${ }^{\mathrm{HEt}}$ in crystalline form following a procedure analogous to that reported by Gusev for the isolation of RuPNN ${ }^{\mathrm{H2}}{ }^{13 \mathrm{C}}$ In an argon glovebox, RuPNN $^{\text {imine }}(316 \mathrm{mg}, 0.450 \mathrm{mmol}$ ) was dissolved in $6.0 \mathrm{~mL}$ toluene in a $40 \mathrm{~mL}$ vial. Then, $30 \mathrm{~mL}$ pentane was added to the vial, and the dark purple solution was briefly swirled to mix. The open vial was placed in a $450 \mathrm{~mL}$ Parr pressure reactor, which was sealed and brought out of the glovebox. The vessel was 
then pressurized with hydrogen to 30 bar and allowed to sit at room temperature for three days without stirring. The pressure vessel was carefully vented, closed (with one atmosphere of hydrogen remaining), brought into the glovebox, and opened. The vial was removed and observed to contain yellow crystals and a pale purple/brown mother liquor. The crystals were collected and washed several times with pentane, then dried under vacuum for only one hour. Yield: $160 \mathrm{mg}, 84 \%$ The product RuPNN ${ }^{\text {HEt }}$ is stable under inert atmosphere in the solid state for several days at room temperature, and is stable for months under inert atmosphere at $-37^{\circ} \mathrm{C}$. However, dissolution in benzene- $\mathrm{d}_{6}$ at room temperature resulted in rapid formation of a brown solution, and the NMR spectrum indicated decomposition. Dissolving in toluene- $d_{8}$ at $-37{ }^{\circ} \mathrm{C}$ and recording NMR spectra at $-30{ }^{\circ} \mathrm{C}$ allowed confirmation that this product matches the sample of RuPNN ${ }^{\mathrm{HEt}}$ previously characterized only in solution under hydrogen pressure. ${ }^{9} \mathrm{X}$ ray crystallography confirmed that the crystalline product contains one half-equivalent of toluene per ruthenium complex, which was consistent with elemental analysis data. Anal. calcd. for $\mathrm{C}_{18} \mathrm{H}_{33} \mathrm{~N}_{2} \mathrm{OPRu} \cdot 1 / 2\left(\mathrm{C}_{7} \mathrm{H}_{8}\right): \mathrm{C}, 54.76 ; \mathrm{H}, 7.91 ; \mathrm{N}, 5.94$. Found: $\mathrm{C}, 54.61 ; \mathrm{H}, 7.71 ; \mathrm{N}, 5.90$.

Synthesis of RuPNN ${ }^{\text {HOEt }}$. RuPNN ${ }^{\text {HOEt }}$ forms rapidly and cleanly when solid RuPNN ${ }^{\text {HEt }}$ is added to ethanol or a solution of ethanol in pentane, benzene or toluene at room temperature. RuPNN ${ }^{\text {HOEt }}$ was characterized by NMR spectroscopy in solution by dissolving RuPNN ${ }^{\mathrm{HEt}}$ ( $30 \mathrm{mg}, 0.071 \mathrm{mmol}$ ) in toluene$\mathrm{d}_{8}(0.600 \mathrm{~mL})$ containing ethanol $(0.020 \mathrm{~mL}, 0.34 \mathrm{mmol})$. Rapid evolution of hydrogen gas was observed, and ${ }^{1} \mathrm{H}$ NMR spectroscopy immediately after preparation showed the formation of one species assigned as RuPNN ${ }^{\text {HOEt }}$. Detailed NMR characterization was conducted at room temperature in $\mathrm{C}_{6} \mathrm{D}_{6}$. At room temperature, bound ethoxide, the $\mathrm{N}-\mathrm{H}$, and one of the $\mathrm{CH}_{2} \mathrm{P}$ hydrogens are in fast exchange with free ethanol. NMR spectra recorded over the range of $-90^{\circ} \mathrm{C}$ to $20^{\circ} \mathrm{C}$, in toluene- $\mathrm{d}_{8}$ showed the decoalescence of these resonances (See Supporting Information for spectral images). ${ }^{1} \mathrm{H} N M R\left(\mathrm{C}_{6} \mathrm{D}_{6}, 25\right.$ $\left.{ }^{\circ} \mathrm{C}\right): \delta 6.92\left(\mathrm{t}, 1 \mathrm{H},{ }^{3} \mathrm{JHH}_{\mathrm{HH}}=7.9 \mathrm{~Hz}, \mathrm{CH}_{\mathrm{pyr}}\right) ; 6.78\left(\mathrm{~d}, 1 \mathrm{H},{ }^{3} J_{\mathrm{HH}}=7.8 \mathrm{~Hz}, \mathrm{CH}_{\mathrm{pyr}}\right) ; 6.41\left(\mathrm{~d}, 1 \mathrm{H},{ }^{3} \mathrm{JHH}_{\mathrm{HH}}=7.7 \mathrm{~Hz}, \mathrm{CH}_{\mathrm{pyr}}\right)$; 3.84 (br s, free EtOH); $3.78\left(\mathrm{dd}, 1 \mathrm{H},{ }^{2} \mathrm{~J}_{\mathrm{HH}}=16.1 \mathrm{~Hz},{ }^{4} \mathrm{~J}_{\mathrm{PH}}=3.7 \mathrm{~Hz}, \mathrm{pyrCH}{ }_{2} \mathrm{~N}\right) ; 3.53\left(\mathrm{br}\right.$, free $\left.\mathrm{CH}_{3} \mathrm{CH}_{2} \mathrm{OH}\right) ; 3.53$ $\left(\mathrm{d}, 1 \mathrm{H},{ }^{2} \mathrm{~J}_{\mathrm{HH}}=16.1 \mathrm{~Hz}, \operatorname{pyrCH} \mathrm{N}\right) ; 3.07\left(\mathrm{brd}, 1 \mathrm{H},{ }^{2} \mathrm{~J}_{\mathrm{HP}}=11.0 \mathrm{~Hz}, \mathrm{CH}_{2} \mathrm{P}\right) ; 2.97\left(\mathrm{~m}, 1 \mathrm{H}, \mathrm{NCH}_{2} \mathrm{CH}_{3}\right) ; 2.84(\mathrm{~m}, 1 \mathrm{H}$, $\left.\mathrm{NCH}_{2} \mathrm{CH}_{3}\right) ; 1.46\left(\mathrm{~d}, 9 \mathrm{H},{ }^{3} \mathrm{HP}_{\mathrm{HP}}=13.4 \mathrm{~Hz}, \mathrm{P}^{\mathrm{t} B u}\right) ; 1.25\left(\mathrm{t}, 3 \mathrm{H},{ }^{3} \mathrm{~J}_{\mathrm{HH}}=7.2 \mathrm{~Hz}, \mathrm{NCH}_{2} \mathrm{CH}_{3}\right) ; 1.10\left(\mathrm{t},{ }^{3} \mathrm{HHH}_{\mathrm{HH}}=7.0 \mathrm{~Hz}\right.$, free $\left.\mathrm{CH}_{3} \mathrm{CH}_{2} \mathrm{OH}\right) ; 1.06\left(\mathrm{~d}, 9 \mathrm{H},{ }^{3} \mathrm{~J}_{\mathrm{HP}}=12.9 \mathrm{~Hz}, \mathrm{P}^{\mathrm{t}} \mathrm{Bu}\right) ;-15.8\left(\mathrm{~d},{ }^{2} J_{\mathrm{HP}}=23.0 \mathrm{~Hz}, \mathrm{Ru}-\mathrm{H}\right) .{ }^{31} \mathrm{P}\left\{{ }^{1 \mathrm{H}}\right\} \mathrm{NMR}\left(\mathrm{C}_{6} \mathrm{D}_{6}, 25^{\circ} \mathrm{C}\right): \delta$ 105.9. ${ }^{13} \mathrm{C} \mathrm{NMR}\left(\mathrm{C}_{6} \mathrm{D}_{6}, 25^{\circ} \mathrm{C}\right): \delta 208.7\left(\mathrm{~d},{ }^{2} \mathrm{~J}_{\mathrm{CP}}=15.8 \mathrm{~Hz}, \mathrm{C} \equiv 0\right) ; 162.1\left(\mathrm{~d},{ }^{2} \mathrm{~J}_{\mathrm{CP}}=4.7 \mathrm{~Hz}, \mathrm{C}_{\text {pyr }}\right) ; 159.7\left(\mathrm{~d},{ }^{4} J_{\mathrm{CP}}=\right.$ $\left.2.2 \mathrm{~Hz}, \mathrm{C}_{\text {pyr }}\right) ; 136.3\left(\mathrm{~s}, \mathrm{C}_{\text {pyr }}\right) ; 120.5$ (d, $\left.{ }^{3} \mathrm{~J}_{\mathrm{CP}}=9.0 \mathrm{~Hz}, \mathrm{C}_{\text {pyr }}\right) ; 117.4$ (s, $\left.\mathrm{C}_{\text {pyr }}\right) 60.2$ (s, pyrCH ${ }_{2} \mathrm{~N}$ ); 58.9 (br s, free $\mathrm{CH}_{3} \mathrm{CH}_{2} \mathrm{OH}$ ); 50.6 (s, $\mathrm{NCH}_{2} \mathrm{CH}_{3}$ ); 36.6 (d, $\left.{ }^{1} \mathrm{~J}_{\mathrm{CP}}=16.5 \mathrm{~Hz}, \mathrm{PC}\left(\mathrm{CH}_{3}\right)_{3}\right) ; 36.6$ (d, ${ }^{1} \mathrm{~J}_{\mathrm{CP}}=21.7 \mathrm{~Hz}, \mathrm{PCH}_{2} \mathrm{pyr}$ ); 36.1 (d, $\left.{ }^{1} J_{\mathrm{CP}}=16.4 \mathrm{~Hz}, \mathrm{PC}\left(\mathrm{CH}_{3}\right)_{3}\right) ; 29.7\left(\mathrm{~d},{ }^{3} J_{\mathrm{CP}}=4.6 \mathrm{HZ}, \mathrm{PC}\left(\mathrm{CH}_{3}\right)_{3}\right) ; 28.2\left(\mathrm{~d},{ }^{3} J_{\mathrm{CP}}=3.6 \mathrm{HZ}, \mathrm{PC}\left(\mathrm{CH}_{3}\right)_{3}\right) ; 19.8$ (br s, free $\mathrm{CH}_{3} \mathrm{CH}_{2} \mathrm{OH}$ ); 14.91 (s, $\mathrm{NCH}_{2} \mathrm{CH}_{3}$ ).

The extremely high solubility of RuPNN ${ }^{\text {HOEt }}$ in polar and nonpolar solvents prevented straightforward isolation in bulk as a solid, but X-ray-quality crystals were obtained in the following manner: In the glovebox, RuPNN ${ }^{\text {HEt }}(20 \mathrm{mg}, 0.047 \mathrm{mmol}$ ) was combined with one drop of ethanol in a small vial. Rapid evolution of hydrogen gas and the formation of a concentrated yellow solution were observed. Most of the ethanol was evaporated to leave a viscous film, after which pentane (1 mL) was added to give a yellow solution. This solution was capped loosely and allowed to slowly evaporate in the glovebox at room temperature. After one day, yellow crystals were observed, which were suitable for crystallographic characterization. Attempts to isolate the solid product obtained in this manner were not successful, as the solid was observed to decompose under vacuum, and to partly decompose giving a blue solution when washed with pentane. Dissolution of this solid in benzene- $d_{6}$ led to rapid decomposition, giving a blue solution containing several unidentified products. The solid product was 
stable when dissolved in benzene- $d_{6}$ containing a small amount of ethanol, and was identical by NMR to samples prepared directly from RuPNN ${ }^{\text {HEt }}$ as described above.

Kinetic Studies. Kinetic experiments were conducted in an Asynt Multicell Parallel High Pressure Reactor, designed to allow sampling of aliquots from five hydrogenation reactions run in parallel. Our customization of this apparatus was described previously. ${ }^{9}$ First, a water bath was set to $27^{\circ} \mathrm{C}$, which we determined consistently gave an internal reactor temperature of between 24.7 and $25.0^{\circ} \mathrm{C}$. The Asynt reactor was brought into the glovebox with oven-dried glass reactor liners and Teflon-coated stir bars. Reaction solutions, with a total volume of $10.0 \mathrm{~mL}$, were prepared with the appropriate amount of RuPNN $^{\text {imine }}$, hexyl hexanoate, 1 -hexanol, and tetradecane ( 0.20 equiv. relative to hexyl hexanoate) as internal standard. The reactor was closed and removed from the glovebox, and allowed to incubate for 20 minutes in the water bath. The hydrogen line was gently purged for 3 minutes, then connected to the reactor. The reactor was filled to 20 bar and vented carefully three times before being filled to the appropriate pressure and marking the start time. Aliquots were removed at predetermined times for analysis by gas chromatography. To ensure that samples represented the reaction mixture without contamination from the transfer line, $0.5 \mathrm{~mL}$ of reaction mixture was discarded before one drop was collected for each aliquot. The concentration of hexyl hexanoate at each time point was determined by integration of its GC signal against the tetradecane standard.

\section{Author Information}

\section{Corresponding Authors}

*jkeith@colgate.edu

*achianese@colgate.edu

\section{ORCID}

Cole E. Jarczyk: 0000-0002-0464-8897

Eamon F. Reynolds: 0000-0002-4850-756X

Sophie. E. Kelly: 0000-0001-8350-3671

Thao Kim: 0000-0003-2342-3455

Tianyi He: 0000-0002-8191-188X

Jason M. Keith: 0000-0002-5292-397X

Anthony R. Chianese: 0000-0002-9140-6115

\section{Supporting Information}

Alternative pathways calculated by DFT and table of computed energies, images of NMR spectra for RuPNN $^{\text {HOEt }}$, details of kinetic modeling (PDF). Atomic coordinates for all computed molecular structures, compiled as one file readable by the free program Mercury ${ }^{42}$ (compiled.xyz). Animations (GIF) for the imaginary vibrational modes of all calculated transition states (TS animations.zip). Copasi model (model.cps) and kinetic data in the format accepted by Copasi (data.txt). 


\section{Acknowledgements}

We thank the National Science Foundation (CHE-1665144 and CHE-1954924) for support of the research project, and (CHE-1726308) for the acquisition of an upgraded NMR spectrometer.

\section{References}

(1) Zhang, J.; Leitus, G.; Ben-David, Y.; Milstein, D. Efficient Homogeneous Catalytic Hydrogenation of Esters to Alcohols. Angew. Chem. Int. Ed. 2006, 45, 1113-1115.

(2) Zhang, J.; Leitus, G.; Ben-David, Y.; Milstein, D. Facile Conversion of Alcohols into Esters and Dihydrogen Catalyzed by New Ruthenium Complexes. J. Am. Chem. Soc. 2005, 127, 1084010841.

(3) (a) Gunanathan, C.; Ben-David, Y.; Milstein, D. Direct Synthesis of Amides from Alcohols and Amines with Liberation of $\mathrm{H}_{2}$. Science 2007, 317, 790-792. (b) Gnanaprakasam, B.; Balaraman, E.; BenDavid, Y.; Milstein, D. Synthesis of Peptides and Pyrazines from Beta-Amino Alcohols through Extrusion of $\mathrm{H}_{2}$ Catalyzed by Ruthenium Pincer Complexes: Ligand-Controlled Selectivity. Angew. Chem. Int. Ed. 2011, 50, 12240-12244. (c) Zeng, H.; Guan, Z. Direct Synthesis of Polyamides Via Catalytic Dehydrogenation of Diols and Diamines. J. Am. Chem. Soc. 2011, 133, 1159-1161. (d) Rigoli, J. W.; Moyer, S. A.; Pearce, S. D.; Schomaker, J. M. Alpha,Beta-Unsaturated Imines Via RuCatalyzed Coupling of Allylic Alcohols and Amines. Org. Biomol. Chem. 2012, 10, 1746-1749. (e) Gnanaprakasam, B.; Milstein, D. Synthesis of Amides from Esters and Amines with Liberation of $\mathrm{H}_{2}$ under Neutral Conditions. J. Am. Chem. Soc. 2011, 133, 1682-1685. (f) Gnanaprakasam, B.; Ben-David, Y.; Milstein, D. Ruthenium Pincer-Catalyzed Acylation of Alcohols Using Esters with Liberation of Hydrogen under Neutral Conditions. Adv. Synth. Catal. 2010, 352, 3169-3173. (g) Balaraman, E.; Gunanathan, C.; Zhang, J.; Shimon, L. J. W.; Milstein, D. Efficient Hydrogenation of Organic Carbonates, Carbamates and Formates Indicates Alternative Routes to Methanol Based on $\mathrm{CO}_{2}$ and CO. Nature Chem. 2011, 3, 609-614. (h) Huff, C. A.; Sanford, M. S. Catalytic $\mathrm{CO}_{2}$ Hydrogenation to Formate by a Ruthenium Pincer Complex. ACS Catal. 2013, 3, 2412-2416. (i) Chaudhari, M. B.; Bisht, G. S.; Kumari, P.; Gnanaprakasam, B. Ruthenium-Catalyzed Direct Alpha-Alkylation of Amides Using Alcohols. Org. Biomol. Chem. 2016, 14, 9215-9220.

(4) (a) Saudan, L. A.; Saudan, C. M.; Debieux, C.; Wyss, P. Dihydrogen Reduction of Carboxylic Esters to Alcohols under the Catalysis of Homogeneous Ruthenium Complexes: High Efficiency and Unprecedented Chemoselectivity. Angew. Chem. Int. Ed. 2007, 46, 7473-7476. (b) Spasyuk, D.; Gusev, D. G. Acceptorless Dehydrogenative Coupling of Ethanol and Hydrogenation of Esters and Imines. Organometallics 2012, 31, 5239-5242. (c) Spasyuk, D.; Smith, S.; Gusev, D. G. Replacing Phosphorus with Sulfur for the Efficient Hydrogenation of Esters. Angew. Chem. Int. Ed. 2013, 52, 2538-2542. (d) Li, W.; Xie, J.-H.; Yuan, M.-L.; Zhou, Q.-L. Ruthenium Complexes of Tetradentate Bipyridine Ligands: Highly Efficient Catalysts for the Hydrogenation of Carboxylic Esters and Lactones. Green Chem. 2014, 16, 4081-4085. (e) Filonenko, G. A.; Aguila, M. J. B.; Schulpen, E. N.; van Putten, R.; Wiecko, J.; Müller, C.; Lefort, L.; Hensen, E. J. M.; Pidko, E. A. Bis$\mathrm{N}$-Heterocyclic Carbene Aminopincer Ligands Enable High Activity in Ru-Catalyzed Ester Hydrogenation. J. Am. Chem. Soc. 2015, 137, 7620-7623. (f) Spasyuk, D.; Vicent, C.; Gusev, D. G. Chemoselective Hydrogenation of Carbonyl Compounds and Acceptorless Dehydrogenative Coupling of Alcohols. J. Am. Chem. Soc. 2015, 137, 3743-3746. (g) Tan, X.; Wang, Q.; Liu, Y.; Wang, F.; Lv, H.; Zhang, X. A New Designed Hydrazine Group-Containing Ruthenium Complex Used for Catalytic Hydrogenation of Esters. Chem. Commun. 2015, 51, 12193-12196. (h) Tan, X.; Wang, Y.; Liu, Y.; Wang, F.; Shi, L.; Lee, K. H.; Lin, Z.; Lv, H.; Zhang, X. Highly Efficient 
Tetradentate Ruthenium Catalyst for Ester Reduction: Especially for Hydrogenation of Fatty Acid Esters. Org. Lett. 2015, 17, 454-457. (i) Wang, Z.; Chen, X.; Liu, B.; Liu, Q.-B.; Solan, G. A.; Yang, X.; Sun, W.-H. Cooperative Interplay between a Flexible PNN-Ru(II) Complex and a $\mathrm{Nabh}_{4}$ Additive in the Efficient Catalytic Hydrogenation of Esters. Catal. Sci. Technol. 2017, 7, 12971304.

(5) (a) O, W. W. N.; Morris, R. H. Ester Hydrogenation Catalyzed by a Ruthenium(II) Complex Bearing an $\mathrm{N}$-Heterocyclic Carbene Tethered with an " $\mathrm{NH}_{2}$ " Group and a DFT Study of the Proposed Bifunctional Mechanism. ACS Catal. 2013, 3, 32-40. (b) Otsuka, T.; Ishii, A.; Dub, P. A.; Ikariya, T. Practical Selective Hydrogenation of Alpha-Fluorinated Esters with Bifunctional Pincer-Type Ruthenium(II) Catalysts Leading to Fluorinated Alcohols or Fluoral Hemiacetals. J. Am. Chem. Soc. 2013, 135, 9600-9603. (c) Chakraborty, S.; Lagaditis, P. O.; Förster, M.; Bielinski, E. A.; Hazari, N.; Holthausen, M. C.; Jones, W. D.; Schneider, S. Well-Defined Iron Catalysts for the Acceptorless Reversible Dehydrogenation-Hydrogenation of Alcohols and Ketones. ACS Catal. 2014, 4, 3994-4003. (d) Chen, T.; Li, H.; Qu, S.; Zheng, B.; He, L.; Lai, Z.; Wang, Z.-X.; Huang, K.-W. Hydrogenation of Esters Catalyzed by Ruthenium $\mathrm{PN}_{3}$-Pincer Complexes Containing an Aminophosphine Arm. Organometallics 2014, 33, 4152-4155. (e) Junge, K.; Wendt, B.; Jiao, H.; Beller, M. Iridium-Catalyzed Hydrogenation of Carboxylic Acid Esters. ChemCatChem 2014, 6, 2810-2814. (f) Qu, S.; Dai, H.; Dang, Y.; Song, C.; Wang, Z.-X.; Guan, H. Computational Mechanistic Study of Fe-Catalyzed Hydrogenation of Esters to Alcohols: Improving Catalysis by Accelerating Precatalyst Activation with a Lewis Base. ACS Catal. 2014, 4, 4377-4388. (g) Werkmeister, S.; Junge, K.; Wendt, B.; Alberico, E.; Jiao, H.; Baumann, W.; Junge, H.; Gallou, F.; Beller, M. Hydrogenation of Esters to Alcohols with a Well-Defined Iron Complex. Angew. Chem. Int. Ed. 2014, 53, 8722-8726. (h) Chen, X.; Jing, Y.; Yang, X. Unexpected Direct Hydride Transfer Mechanism for the Hydrogenation of Ethyl Acetate to Ethanol Catalyzed by Sns Pincer Ruthenium Complexes. Chem. Eur. J. 2016, 22, 1950-1957. (i) Gusev, D. G. Dehydrogenative Coupling of Ethanol and Ester Hydrogenation Catalyzed by Pincer-Type YNP Complexes. ACS Catal. 2016, 6, 6967-6981. (j) van Putten, R.; Uslamin, E. A.; Garbe, M.; Liu, C.; Gonzalez-deCastro, A.; Lutz, M.; Junge, K.; Hensen, E. J. M.; Beller, M.; Lefort, L.; Pidko, E. A. Non-Pincer-Type Manganese Complexes as Efficient Catalysts for the Hydrogenation of Esters. Angew. Chem. Int. Ed. 2017, 56, 7531-7534.

(6) (a) Dub, P. A.; Henson, N. J.; Martin, R. L.; Gordon, J. C. Unravelling the Mechanism of the Asymmetric Hydrogenation of Acetophenone by [RuX ${ }_{2}$ (Diphosphine)(1,2-Diamine)] Catalysts. J. Am. Chem. Soc. 2014, 136, 3505-3521. (b) Dub, P. A.; Gordon, J. C. The Mechanism of Enantioselective Ketone Reduction with Noyori and Noyori-Ikariya Bifunctional Catalysts. Dalton Trans. 2016, 45, 6756-6781. (c) Dub, P. A.; Scott, B. L.; Gordon, J. C. Why Does Alkylation of the $\mathrm{N}-\mathrm{H}$ Functionality within M/NH Bifunctional Noyori-Type Catalysts Lead to Turnover? J. Am. Chem. Soc. 2017, 139, 1245-1260. (d) Tindall, D. J.; Menche, M.; Schelwies, M.; Paciello, R. A.; Schafer, A.; Comba, P.; Rominger, F.; Hashmi, A. S. K.; Schaub, T. Ru(0) or Ru(II): A Study on Stabilizing the "Activated" Form of Ru-PNP Complexes with Additional Phosphine Ligands in Alcohol Dehydrogenation and Ester Hydrogenation. Inorg. Chem. 2020, 59, 5099-5115.

(7) (a) del Pozo, C.; Iglesias, M.; Sánchez, F. I. Pincer-Type Pyridine-Based N-Heterocyclic Carbene Amine $\mathrm{Ru}$ (II) Complexes as Efficient Catalysts for Hydrogen Transfer Reactions. Organometallics 2011, 30, 2180-2188. (b) Sun, Y.; Koehler, C.; Tan, R.; Annibale, V. T.; Song, D. Ester Hydrogenation Catalyzed by Ru-CNN Pincer Complexes. Chem. Commun. 2011, 47, 8349-8351. (c) Kim, D.; Le, L.; Drance, M. J.; Jensen, K. H.; Bogdanovski, K.; Cervarich, T. N.; Barnard, M. G.; Pudalov, N. J.; Knapp, S. M. M.; Chianese, A. R. Ester Hydrogenation Catalyzed by CNN-Pincer Complexes of Ruthenium. Organometallics 2016, 35, 982-989. (d) Le, L.; Liu, J.; He, T.; Kim, D.; Lindley, E. J.; Cervarich, T. N.; Malek, J. C.; Pham, J.; Buck, M. R.; Chianese, A. R. Structure-Function 
Relationship in Ester Hydrogenation Catalyzed by Ruthenium CNN-Pincer Complexes. Organometallics 2018, 37, 3286-3297.

(8) Le, L.; Liu, J.; He, T.; Malek, J. C.; Cervarich, T. N.; Buttner, J. C.; Pham, J.; Keith, J. M.; Chianese, A. R. Unexpected CNN-to-CC Ligand Rearrangement in Pincer-Ruthenium Precatalysts Leads to a Base-Free Catalyst for Ester Hydrogenation. Organometallics 2019, 38, 3311-3321.

(9) He, T.; Buttner, J. C.; Reynolds, E. F.; Pham, J.; Malek, J. C.; Keith, J. M.; Chianese, A. R. Dehydroalkylative Activation of CNN- and PNN-Pincer Ruthenium Catalysts for Ester Hydrogenation. J. Am. Chem. Soc. 2019, 141, 17404-17413.

(10) Fogler, E.; Garg, J. A.; Hu, P.; Leitus, G.; Shimon, L. J. W.; Milstein, D. System with Potential Dual Modes of Metal-Ligand Cooperation: Highly Catalytically Active Pyridine-Based PNNH-Ru Pincer Complexes. Chem. Eur. J. 2014, 20, 15727-15731.

(11) (a) Takebayashi, S.; Bergens, S. H. Facile Bifunctional Addition of Lactones and Esters at Low Temperatures. The First Intermediates in Lactone/Ester Hydrogenations. Organometallics 2009, 28, 2349-2351. (b) Carpenter, I.; Eckelmann, S. C.; Kuntz, M. T.; Fuentes, J. A.; France, M. B.; Clarke, M. L. Convenient and Improved Protocols for the Hydrogenation of Esters Using Ru Catalysts Derived from (P,P), (P,N,N) and (P,N,O) Ligands. Dalton Trans. 2012, 41, 10136-10140. (c) Fuentes, J. A.; Smith, S. M.; Scharbert, M. T.; Carpenter, I.; Cordes, D. B.; Slawin, A. M. Z.; Clarke, M. L. On the Functional Group Tolerance of Ester Hydrogenation and Polyester Depolymerisation Catalysed by Ruthenium Complexes of Tridentate Aminophosphine Ligands. Chem. Eur. J. 2015, 21, 10851-10860. (d) Ogata, O.; Nakayama, Y.; Nara, H.; Fujiwhara, M.; Kayaki, Y. Atmospheric Hydrogenation of Esters Catalyzed by PNP-Ruthenium Complexes with an N-Heterocyclic Carbene Ligand. Org. Lett. 2016, 18, 3894-3897.

(12) (a) Kuriyama, W.; Ino, Y.; Ogata, O.; Sayo, N.; Saito, T. A Homogeneous Catalyst for Reduction of Optically Active Esters to the Corresponding Chiral Alcohols without Loss of Optical Purities. Adv. Synth. Catal. 2010, 352, 92-96. (b) Zhang, J.; Balaraman, E.; Leitus, G.; Milstein, D. Electron-Rich PNP- and PNN-Type Ruthenium(II) Hydrido Borohydride Pincer Complexes. Synthesis, Structure, and Catalytic Dehydrogenation of Alcohols and Hydrogenation of Esters. Organometallics 2011, 30, 5716-5724. (c) Spasyuk, D.; Smith, S.; Gusev, D. G. From Esters to Alcohols and Back with Ruthenium and Osmium Catalysts. Angew. Chem. Int. Ed. 2012, 51, 2772-2775. (d) Ziebart, C.; Jackstell, R.; Beller, M. Selective Catalytic Hydrogenation of Diethyl Oxalate and Related Esters. Chem CatChem 2013, 5, 3228-3231. (e) Chakraborty, S.; Dai, H.; Bhattacharya, P.; Fairweather, N. T.; Gibson, M. S.; Krause, J. A.; Guan, H. Iron-Based Catalysts for the Hydrogenation of Esters to Alcohols. J. Am. Chem. Soc. 2014, 136, 7869-7872. (f) vom Stein, T.; Meuresch, M.; Limper, D.; Schmitz, M.; Holscher, M.; Coetzee, J.; Cole-Hamilton, D. J.; Klankermayer, J.; Leitner, W. Highly Versatile Catalytic Hydrogenation of Carboxylic and Carbonic Acid Derivatives Using a Ru-Triphos Complex: Molecular Control over Selectivity and Substrate Scope. J. Am. Chem. Soc. 2014, 136, 13217-13225. (g) Korstanje, T. J.; van der Vlugt, J. I.; Elsevier, C. J.; de Bruin, B. Hydrogenation of Carboxylic Acids with a Homogeneous Cobalt Catalyst. Science 2015, 350, 298-302. (h) Brewster, T. P.; Rezayee, N. M.; Culakova, Z.; Sanford, M. S.; Goldberg, K. I. Base-Free Iridium-Catalyzed Hydrogenation of Esters and Lactones. ACS Catal. 2016, 6, 3113-3117. (i) Espinosa-Jalapa, N. A.; Nerush, A.; Shimon, L. J. W.; Leitus, G.; Avram, L.; Ben-David, Y.; Milstein, D. ManganeseCatalyzed Hydrogenation of Esters to Alcohols. Chem. Eur. J. 2017, 23, 5934-5938. (j) Yuwen, J.; Chakraborty, S.; Brennessel, W. W.; Jones, W. D. Additive-Free Cobalt-Catalyzed Hydrogenation of Esters to Alcohols. ACS Catal. 2017, 7, 3735-3740. (k) Anaby, A.; Schelwies, M.; Schwaben, J.; Rominger, F.; Hashmi, A. S. K.; Schaub, T. Study of Precatalyst Degradation Leading to the Discovery of a New $\mathrm{Ru}^{0}$ Precatalyst for Hydrogenation and Dehydrogenation. Organometallics 2018, 37, 2193-2201. 
(13) (a) Li, H.; Wang, X.; Huang, F.; Lu, G.; Jiang, J.; Wang, Z.-X. Computational Study on the Catalytic Role of Pincer Ruthenium(II)-PNN Complex in Directly Synthesizing Amide from Alcohol and Amine: The Origin of Selectivity of Amide over Ester and Imine. Organometallics 2011, 30, 5233-5247. (b) Wang, H.; Liu, C.; Zhang, D. Decisive Effects of Solvent and Substituent on the Reactivity of Ru-Catalyzed Hydrogenation of Ethyl Benzoate to Benzyl Alcohol and Ethanol: A DFT Study. Mol. Catal. 2017, 440, 120-132. (c) Gusev, D. G. Revised Mechanisms of the Catalytic Alcohol Dehydrogenation and Ester Reduction with the Milstein PNN Complex of Ruthenium. Organometallics 2020, 39, 258-270.

(14) (a) Zeng, G.; Li, S. Insights into Dehydrogenative Coupling of Alcohols and Amines Catalyzed by a (PNN)-Ru(II) Hydride Complex: Unusual Metal-Ligand Cooperation. Inorg. Chem. 2011, 50, 10572-10580. (b) Li, H.; Wen, M.; Wang, Z.-X. Computational Mechanistic Study of the Hydrogenation of Carbonate to Methanol Catalyzed by the (RuPNN)-P-II Complex. Inorg. Chem. 2012, 51, 5716-5727. (c) Yang, X. Metal Hydride and Ligand Proton Transfer Mechanism for the Hydrogenation of Dimethyl Carbonate to Methanol Catalyzed by a Pincer Ruthenium Complex. ACS Catal. 2012, 2, 964-970. (d) Cho, D.; Ko, K. C.; Lee, J. Y. Catalytic Mechanism for the Ruthenium-Complex-Catalyzed Synthesis of Amides from Alcohols and Amines: A DFT Study. Organometallics 2013, 32, 4571-4576. (e) Hasanayn, F.; Baroudi, A.; Bengali, A. A.; Goldman, A. S. Hydrogenation of Dimethyl Carbonate to Methanol by Trans-[Ru(H) $\left.)_{2}(\mathrm{PNN})(\mathrm{CO})\right]$ Catalysts: DFT Evidence for Ion-Pair-Mediated Metathesis Paths for C-OMe Bond Cleavage. Organometallics 2013, 32, 6969-6985. (f) Hasanayn, F.; Harb, H. A Metathesis Model for the Dehydrogenative Coupling of Amines with Alcohols and Esters into Carboxamides by Milstein's [Ru(PNN)(CO)(H)] Catalysts. Inorg. Chem. 2014, 53, 8334-8349. (g) Li, L.; Lei, M.; Liu, L.; Xie, Y.; Schaefer, H. F. Metal-Substrate Cooperation Mechanism for Dehydrogenative Amidation Catalyzed by a PNNRu Catalyst. Inorg. Chem. 2018, 57, 8778-8787. (h) Shu, S.; Huang, M.; Jiang, J.; Qu, L.-B.; Liu, Y.; Ke, Z. Catalyzed or Non-Catalyzed: Chemoselectivity of Ru-Catalyzed Acceptorless Dehydrogenative Coupling of Alcohols and Amines Via Metal-Ligand Bond Cooperation and (De) Aromatization. Catal. Sci. Technol. 2019, 9, 2305-2314.

(15) Kozuch, S.; Shaik, S. How to Conceptualize Catalytic Cycles? The Energetic Span Model. Acc. Chem. Res. 2010, 44, 101-110.

(16) (a) Kubis, C.; Sawall, M.; Block, A.; Neymeyr, K.; Ludwig, R.; Borner, A.; Selent, D. An Operando FTIR Spectroscopic and Kinetic Study of Carbon Monoxide Pressure Influence on Rhodium-Catalyzed Olefin Hydroformylation. Chemistry 2014, 20, 11921-31. (b) Szlapa, E. N.; Harvey, J. N. Computational Modelling of Selectivity in Cobalt-Catalyzed Propene Hydroformylation. Chem. Eur. J. 2018, 24, 17096-17104. (c) Brezny, A. C.; Landis, C. R. Development of a Comprehensive Microkinetic Model for Rh(Bis(Diazaphospholane))-Catalyzed Hydroformylation. ACS Catal. 2019, 9, 2501-2513.

(17) (a) Nakatsuka, H.; Yamamura, T.; Shuto, Y.; Tanaka, S.; Yoshimura, M.; Kitamura, M. Mechanism of Asymmetric Hydrogenation of Aromatic Ketones Catalyzed by a Combined System of Ru(Pi$\left.\mathrm{CH}_{2} \mathrm{C}\left(\mathrm{CH}_{3}\right) \mathrm{CH}_{2}\right)_{2}(\mathrm{cod})$ and the Chiral $\mathrm{sp}^{2} \mathrm{~N} / \mathrm{sp}^{3} \mathrm{NH}$ Hybrid Linear N4 Ligand Ph-BINAN-H-Py. J. Am. Chem. Soc. 2015, 137, 8138-8149. (b) Nakane, S.; Yamamura, T.; Manna, S. K.; Tanaka, S.; Kitamura, M. Mechanistic Study of the Ru-Catalyzed Asymmetric Hydrogenation of Nonchelatable and Chelatable Tert-Alkyl Ketones Using the Linear Tridentate $\mathrm{sp}^{3} \mathrm{P} / \mathrm{sp}^{3} \mathrm{NH} / \mathrm{sp}^{2} \mathrm{~N}$ Combined Ligand Pn(H)N: Runh- and Runk-Involved Dual Catalytic Cycle. ACS Catal. 2018, 8, 11059-11075.

(18) Noyori, R.; Ohkuma, T. Asymmetric Catalysis by Architectural and Functional Molecular Engineering: Practical Chemo- and Stereoselective Hydrogenation of Ketones. Angew. Chem. Int. Ed. 2001, 40, 40-73. 
(19) (a) Yang, X.; Hall, M. B. Mechanism of Water Splitting and Oxygen-Oxygen Bond Formation by a Mononuclear Ruthenium Complex. J. Am. Chem. Soc. 2010, 132, 120-130. (b) Sandhya, K. S.; Suresh, C. H. Water Splitting Promoted by a Ruthenium(II) PNN Complex: An Alternate Pathway through a Dihydrogen Complex for Hydrogen Production. Organometallics 2011, 30, 3888-3891. (c) Ma, C.; Piccinin, S.; Fabris, S. Reaction Mechanisms of Water Splitting and $\mathrm{H}_{2}$ Evolution by a $\mathrm{Ru}(\mathrm{II})$-Pincer Complex Identified with Ab Initio Metadynamics Simulations. ACS Catal. 2012, 2, 1500-1506. (d) Sandhya, K. S.; Remya, G. S.; Suresh, C. H. Pincer Ligand Modifications to Tune the Activation Barrier for $\mathrm{H}_{2}$ Elimination in Water Splitting Milstein Catalyst. Inorg Chem 2015, 54, 11150-11156.

(20) In several cases, transition states were calculated to have a lower free energy than a connected intermediate species. In each case, we have verified that the intermediate is lower in energy on the optimzed electronic energy surface. See the Computational Methods section for details.

(21) Hasanayn, F.; Baroudi, A. Direct H/OR and OR/OR' Metathesis Pathways in Ester Hydrogenation and Transesterification by Milstein's Catalyst. Organometallics 2013, 32, 2493-2496.

(22) Dawe, L. N.; Karimzadeh-Younjali, M.; Dai, Z.; Khaskin, E.; Gusev, D. G. The Milstein Bipyridyl PNN Pincer Complex of Ruthenium Becomes a Noyori-Type Catalyst under Reducing Conditions. J. Am. Chem. Soc. 2020, 142, 19510-19522.

(23) Smith, N. E.; Bernskoetter, W. H.; Hazari, N. The Role of Proton Shuttles in the Reversible Activation of Hydrogen Via Metal-Ligand Cooperation. J. Am. Chem. Soc. 2019, 141, 17350-17360.

(24) Kumar, A.; von Wolff, N.; Rauch, M.; Zou, Y. Q.; Shmul, G.; Ben-David, Y.; Leitus, G.; Avram, L.; Milstein, D. Hydrogenative Depolymerization of Nylons. J. Am. Chem. Soc. 2020, 142, 1426714275.

(25) Hoops, S.; Sahle, S.; Gauges, R.; Lee, C.; Pahle, J.; Simus, N.; Singhal, M.; Xu, L.; Mendes, P.; Kummer, U. Copasi-a Complex Pathway Simulator. Bioinformatics 2006, 22, 3067-3074.

(26) (a) Artús Suàrez, L.; Culakova, Z.; Balcells, D.; Bernskoetter, W. H.; Eisenstein, O.; Goldberg, K. I.; Hazari, N.; Tilset, M.; Nova, A. The Key Role of the Hemiaminal Intermediate in the IronCatalyzed Deaminative Hydrogenation of Amides. ACS Catal. 2018, 8, 8751-8762. (b) Yu, Y.; Zhu, Y.; Bhagat, M. N.; Raghuraman, A.; Hirsekorn, K. F.; Notestein, J. M.; Nguyen, S. T.; Broadbelt, L. J. Mechanism of Regioselective Ring-Opening Reactions of 1,2-Epoxyoctane Catalyzed by Tris(Pentafluorophenyl)Borane: A Combined Experimental, Density Functional Theory, and Microkinetic Study. ACS Catal. 2018, 8, 11119-11133. (c) Jaraíz, M.; Rubio, J. E.; Enríquez, L.; Pinacho, R.; López-Pérez, J. L.; Lesarri, A. An Efficient Microkinetic Modeling Protocol: Start with Only the Dominant Mechanisms, Adjust All Parameters, and Build the Complete Model Incrementally. ACS Catal. 2019, 9, 4804-4809. (d) Rogers, J. A.; Popp, B. V. Operando Infrared Spectroscopy Study of Iron-Catalyzed Hydromagnesiation of Styrene: Explanation of Nonlinear Catalyst and Inhibitory Substrate Dependencies. Organometallics 2019, 38, 4533-4538. (e) Perez-Soto, R.; Besora, M.; Maseras, F. The Challenge of Reproducing with Calculations Raw Experimental Kinetic Data for an Organic Reaction. Org Lett 2020, 22, 2873-2877. (f) Green, K.A.; Hoover, J. M. Intermediacy of Copper(I) under Oxidative Conditions in the Aerobic CopperCatalyzed Decarboxylative Thiolation of Benzoic Acids. ACS Catal. 2019, 10, 1769-1782.

(27) Brunner, E. Solubility of Hydrogen in 10 Organic Solvents at 298.15, 323.15, and 373.15 K. J. Chem. Eng. Data 1985, 30, 269-273.

(28) (a) Kretschmer, C. B.; Wiebe, R. Liquid-Vapor Equilibrium of Ethanol-Toluene Solutions. J. Am. Chem. Soc. 1949, 71, 1793-1797. (b) Guzman, J.; Teja, A.; Kay, W. Vapor-Liquid Equilibria in Binary Mixtures Formed from Hexamethyldisiloxane, Toluene and Ethanol. Fluid Phase Equilib. 1981, 7, 187-195. 
(29) Xu, Y.; Li, H.; Wang, C.; Ma, L.; Han, S. Prediction of Vapor- Liquid Equilibria of AlcoholHydrocarbon Systems by ${ }^{1} \mathrm{H}$ NMR and Activity Coefficients at Infinite Dilution. Ind. Eng. Chem. Res. 2005, 44, 408-415.

(30) (a) Morris, S. A.; Gusev, D. G. Rethinking the Claisen-Tishchenko Reaction. Angew. Chem. Int. Ed. 2017, 56, 6228-6231. (b) Gusev, D. G.; Spasyuk, D. M. Revised Mechanisms for Aldehyde Disproportionation and the Related Reactions of the Shvo Catalyst. ACS Catal. 2018, 8, 68516861.

(31) Balaraman, E.; Gnanaprakasam, B.; Shimon, L. J. W.; Milstein, D. Direct Hydrogenation of Amides to Alcohols and Amines under Mild Conditions. J. Am. Chem. Soc. 2010, 132, 16756-16758.

(32) Frisch, M. J.; Trucks, G. W.; Schlegel, H. B.; Scuseria, G. E.; Robb, M. A.; Cheeseman, J. R.; Scalmani, G.; Barone, V.; Petersson, G. A.; Nakatsuji, H.; Li, X.; Caricato, M.; Marenich, A. V.; Bloino, J.; Janesko, B. G.; Gomperts, R.; Mennucci, B.; Hratchian, H. P.; Ortiz, J. V.; Izmaylov, A. F.; Sonnenberg, J. L.; Williams; Ding, F.; Lipparini, F.; Egidi, F.; Goings, J.; Peng, B.; Petrone, A.; Henderson, T.; Ranasinghe, D.; Zakrzewski, V. G.; Gao, J.; Rega, N.; Zheng, G.; Liang, W.; Hada, M.; Ehara, M.; Toyota, K.; Fukuda, R.; Hasegawa, J.; Ishida, M.; Nakajima, T.; Honda, Y.; Kitao, O.; Nakai, H.; Vreven, T.; Throssell, K.; Montgomery Jr., J. A.; Peralta, J. E.; Ogliaro, F.; Bearpark, M. J.; Heyd, J. J.; Brothers, E. N.; Kudin, K. N.; Staroverov, V. N.; Keith, T. A.; Kobayashi, R.; Normand, J.; Raghavachari, K.; Rendell, A. P.; Burant, J. C.; Iyengar, S. S.; Tomasi, J.; Cossi, M.; Millam, J. M.; Klene, M.; Adamo, C.; Cammi, R.; Ochterski, J. W.; Martin, R. L.; Morokuma, K.; Farkas, O.; Foresman, J. B.; Fox, D. J. Gaussian 16 Rev. B.01, Wallingford, CT, 2016.

(33) (a) Lee, C.; Yang, W.; Parr, R. G. Development of the Colle-Salvetti Correlation-Energy Formula into a Functional of the Electron Density. Phys. Rev. B 1988, 37, 785-789. (b) Becke, A. D. DensityFunctional Thermochemistry. III. The Role of Exact Exchange. J. Chem. Phys. 1993, 98, 56485652.

(34) Hay, P. J.; Wadt, W. R. Ab Initio Effective Core Potentials for Molecular Calculations. Potentials for K to Au Including the Outermost Core Orbitals. J. Chem. Phys. 1985, 82, 299-310.

(35) Ehlers, A.; Böhme, M.; Dapprich, S.; Gobbi, A.; Höllwarth, A.; Jonas, V.; Köhler, K.; Stegmann, R.; Veldkamp, A.; Frenking, G. A Set of F-Polarization Functions for Pseudo-Potential Basis Sets of the Transition Metals Sc-Cu, Y-Ag and La-Au. Chem. Phys. Lett. 1993, 208, 111-114.

(36) Roy, L. E.; Hay, P. J.; Martin, R. L. Revised Basis Sets for the LANL Effective Core Potentials. J. Chem. Theory Comput. 2008, 4, 1029-1031.

(37) (a) Krishnan, R.; Binkley, J. S.; Seeger, R.; Pople, J. A. Self-Consistent Molecular Orbital Methods. XX. A Basis Set for Correlated Wave Functions. J. Chem. Phys. 1980, 72, 650-654. (b) McLean, A. D.; Chandler, G. S. Contracted Gaussian Basis Sets for Molecular Calculations. I. Second Row Atoms, $\mathrm{Z}=11-18$. J. Chem. Phys. 1980, 72, 5639-5648.

(38) Marenich, A. V.; Cramer, C. J.; Truhlar, D. G. Universal Solvation Model Based on Solute Electron Density and on a Continuum Model of the Solvent Defined by the Bulk Dielectric Constant and Atomic Surface Tensions. J. Phys. Chem. B 2009, 113, 6378-6396.

(39) (a) Zweifel, T.; Naubron, J. V.; Buttner, T.; Ott, T.; Grützmacher, H. Ethanol as Hydrogen Donor: Highly Efficient Transfer Hydrogenations with Rhodium(I) Amides. Angew. Chem. Int. Ed. 2008, 47, 3245-3249. (b) Zweifel, T.; Naubron, J. V.; Grützmacher, H. Catalyzed Dehydrogenative Coupling of Primary Alcohols with Water, Methanol, or Amines. Angew. Chem. Int. Ed. 2009, 48, 559-563. (c) Prokopchuk, D. E.; Morris, R. H. Inner-Sphere Activation, Outer-Sphere Catalysis: Theoretical Study on the Mechanism of Transfer Hydrogenation of Ketones Using Iron(II) PNNP Eneamido Complexes. Organometallics 2012, 31, 7375-7385.

(40) Cramer, C. J., In Essentials of Computational Chemistry, 2nd ed.; Wiley: Chichester, UK, 2004; 378379. 
(41) (a) Zhao, Y.; Truhlar, D. G. The M06 Suite of Density Functionals for Main Group Thermochemistry, Thermochemical Kinetics, Noncovalent Interactions, Excited States, and Transition Elements:

Two New Functionals and Systematic Testing of Four M06-Class Functionals and 12 Other Functionals. Theor. Chem. Acc. 2007, 120, 215-241. (b) Zhao, Y.; Truhlar, D. G. Density Functionals with Broad Applicability in Chemistry. Acc. Chem. Res. 2008, 41, 157-167.

(42) Macrae, C. F.; Edgington, P. R.; McCabe, P.; Pidcock, E.; Shields, G. P.; Taylor, R.; Towler, M.; van de Streek, J. Mercury: Visualization and Analysis of Crystal Structures. J. Appl. Cryst. 2006, 39, 453457.

Graphic for Table of Contents use only:

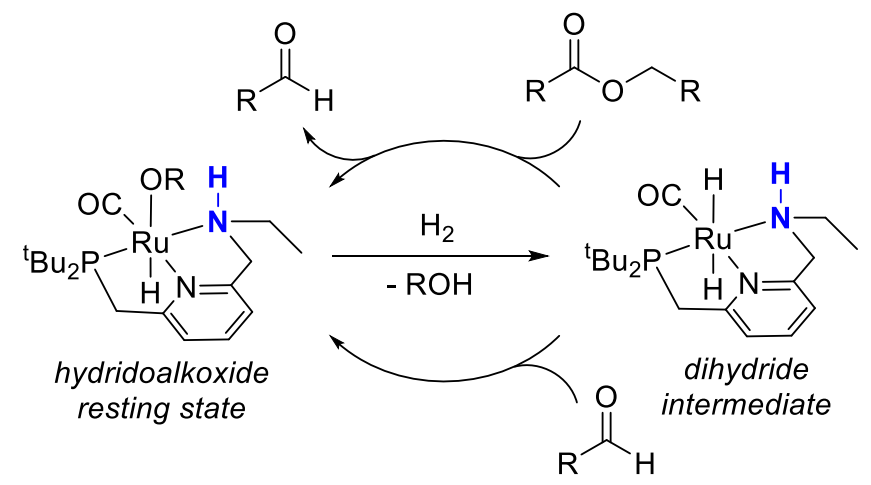

- Density Functional Theory

- Isolation of Intermediates

- Detailed Kinetic Study 